\title{
Application of the Synchronized B-Grid Staggering for Solution of the Shallow-Water Equations on the Spherical Icosahedral Grid
}

\author{
HIROAKI MIURA \\ Department of Earth and Planetary Science, Graduate School of Science, The University of Tokyo, Tokyo, Japan
}

(Manuscript received 27 August 2018, in final form 14 April 2019)

\begin{abstract}
A shallow-water model using the hexagonal synchronized B grid (SB grid) is developed on the spherical icosahedral grid. The SB grid adopts the same variable arrangement as the ZM grid, but does not suffer from a computational mode problem of the ZM grid since interactions in the extra degrees of freedom of velocity fields through the nonlinear terms are excluded. For better representations of the geostrophic balance, a quadratic reconstruction of fluid height inside hexagonal/pentagonal cells is used to configure the gradient with the second-order accuracy. When nongeostrophic motions are more dominant than geostrophic ones, smaller-scale noises arise. To prevent a decoupling of the velocity fields, a hyperviscosity is added to force velocities adjacent to each other to evolve synchronously. Some standard tests are performed to examine the SB-grid shallow-water model. The model is almost second-order accurate if both the initial conditions and the surface topography are smooth and if the influence of the hyperviscosity is small. The SB-grid model is superior to a C-grid model regarding the convergence of error norms in a steady-state geostrophically balanced flow test, while it is inferior to that concerning conservation of total energy in a case of flow over an isolated mountain. An advantage of the SB-grid model is that both accuracy and stability are weakly sensitive to whether a grid optimization is applied or not. The SB grid is an attractive alternative to the conventional A grid and is competitive with the $\mathrm{C}$ grid on the spherical icosahedral grid.
\end{abstract}

\section{Introduction}

Application of the spherical icosahedral grid to the atmospheric modeling has been studied for near half-century after both Sadourny et al. (1968) and Williamson (1968) proposed a method to integrate the barotropic vorticity equation on a hexagonalpentagonal mesh. Williamson (1970) further proceeded to the integration of the shallow-water equations in the momentum form. Tomita et al. (2001) followed the work of Williamson (1970) in developing their hexagonal A-grid model. They improved the accuracy of the model by the following procedures: introducing a grid optimization by the so-called spring dynamics method (Tomita et al. 2002), defining the cell averages at the centroids of hexagonal or pentagonal cells, and switching from Voronoi polygons to barycentric ones (Fig. 1). The reason for the change of the polygon type is that distortion of hexagonal cells is smaller for the barycentric mesh than for the Voronoi mesh (Miura and Kimoto 2005). Masuda and Ohnishi (1986) used

Corresponding author: Hiroaki Miura, h_miura@eps.s.u-tokyo.ac.jp the vorticity-divergence form of the shallow-water equations, which is often called as the hexagonal $\mathrm{Z}$ grid (Heikes and Randall 1995), to conduct a 100-day simulation of the Rossby-Haurwitz wave. Ringler et al. (2010) developed a hexagonal C-grid model based on the so-called TRiSK scheme (Thuburn et al. 2009) that settled a nonstationary geostrophic mode problem of the hexagonal $\mathrm{C}$ grid (Ničković et al. 2002). Bonaventura and Ringler (2005) developed a shallowwater model using the triangular $\mathrm{C}$ grid, and Rípodas et al. (2009) improved it.

In the Dynamical Core Model Intercomparison Project in 2016 (Ullrich et al. 2017), 6 out of 11 threedimensional atmospheric dynamical cores used the spherical icosahedral grid. The horizontal staggerings of those models were as follows: the hexagonal A grid for the Nonhydrostatic Icosahedral Atmosphere Model (NICAM; Satoh et al. 2008, 2014), the hexagonal $\mathrm{Z}$ grid for the Colorado State University model (CSU; Heikes and Randall 1995), the hexagonal C grid for the Model for Prediction Across Scales (MPAS; Skamarock et al. 2012), the Ocean-Land-Atmosphere Model (OLAM; Walko and Avissar 2008a,b), the Dynamical 

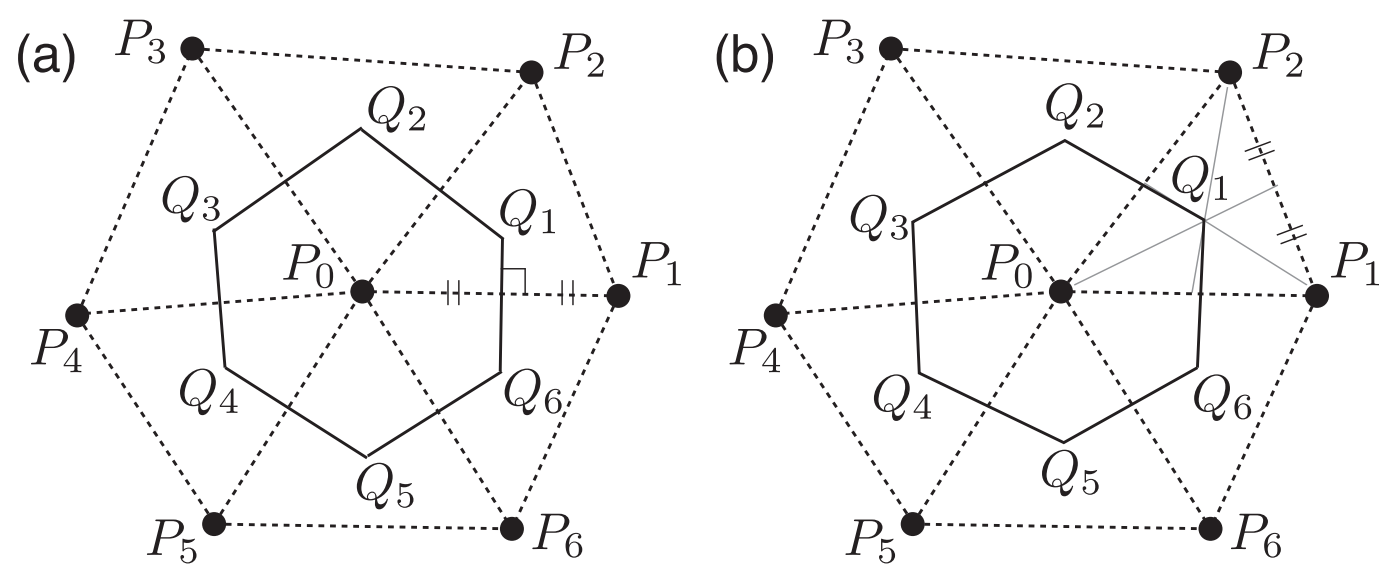

FIG. 1. Schematic illustrations of a hexagonal cell in (a) a Voronoi mesh and (b) a barycentric mesh. The triangular meshes shown by the dotted lines are common.

Core on the Icosahedron (DYNAMICO; Dubos et al. 2015), and the triangular $C$ grid for the Icosahedral Nonhydrostatic model (ICON; Zängl et al. 2015).

Models that participated in the DCMIP2016 used the A, C, or Z grid, which was initially defined for the rectangular mesh (Arakawa and Lamb 1977; Randall 1994) and has been extended to the hexagonal mesh. On the other hand, no model adopted an extension of the Arakawa B grid, which places mass at the centers and velocity vector at the corners of rectangular cells, respectively. Ničković et al. (2002) analyzed the HB/E 1 and HB/E 2 grid (Mesinger 2000) that locates depth at the centers and velocity vector at every other corner of hexagons; we denote them as the $\mathrm{B} 1$ and $\mathrm{B} 2$ grid in this work (Figs. 2b,c). Ničković et al. (2002) found that those grids falsely generate a nonstationary geostrophic solution and nonsymmetric inertia-gravity waves. Ringler and Randall (2002a,b) proposed the ZM grid (Fig. 2a), which arranges velocity vector at every corner of hexagons, and showed that it possesses dispersion properties of the inertia-gravity waves as good as the $\mathrm{Z}$ grid. They also derived a form of the gradient operator at the corners of hexagons that conserves total energy to the magnitude of the machine epsilon (Ringler and Randall 2002a). Danilov et al. (2017) developed a three-dimensional (3D) ocean model using a similar approach as the ZM grid, which was called as the cell-vertex discretization in their paper. Besides those desirable behaviors, the ZM grid has a disadvantage that it supports a computational mode due to the mismatch of the number of the mass points and that of the velocity points (Ringler and Randall 2002b). The ratio of the degrees of freedom of the velocity components to those of the depth is four for the ZM grid, but it must be two ideally.

The above mentioned problem due to the redundancy of velocity of the $\mathrm{ZM}$ grid would be minor if we were able to control it harmlessly. Here, we examine several results of a ZM-grid shallow-water model tested on a centroidal Voronoi mesh, which is generated by (a) SB/ZM

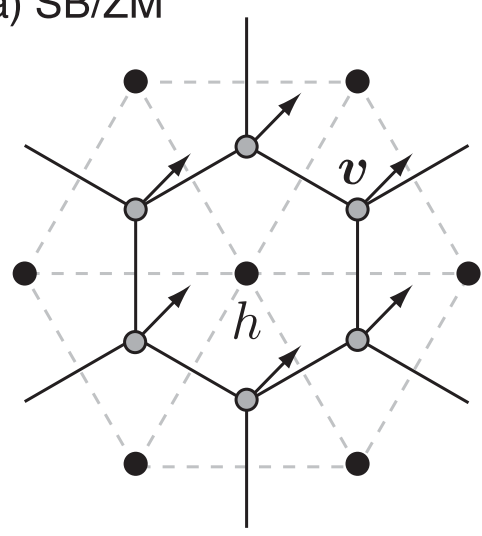

(b) B1

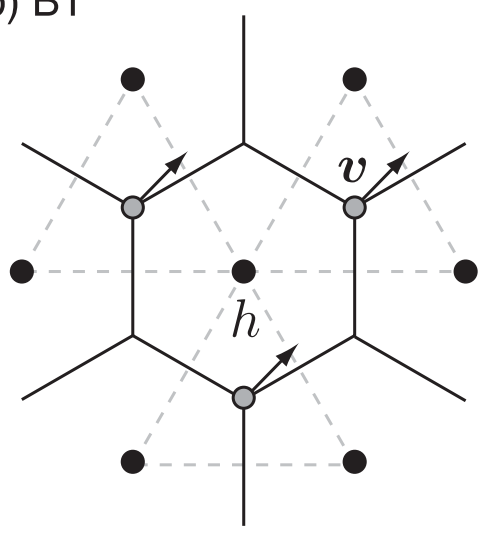

(c) $\mathrm{B} 2$

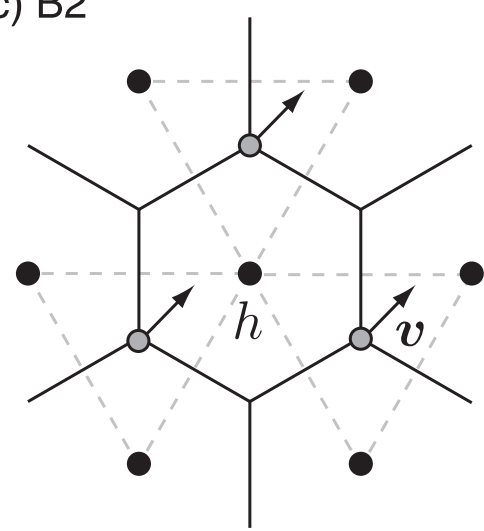

FIG. 2. Schematic illustrations of the arrangements of the prognostic variables. (a) The SB or ZM grid, (b) the B1 grid, and (c) the B2 grid. 


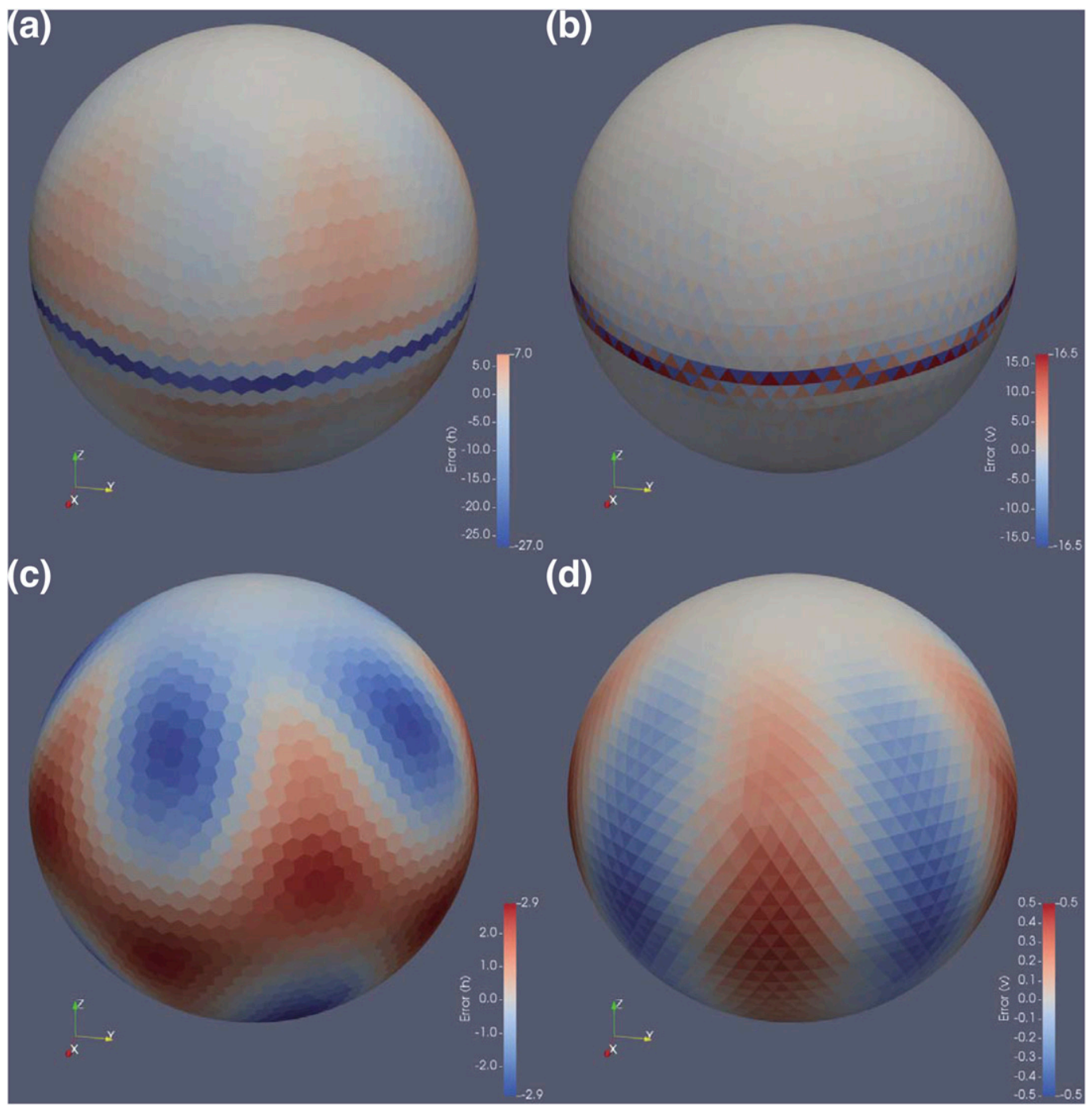

FIG. 3. The test case 2 of Williamson et al. (1992) performed by a ZM-grid model for the grid with 2562 hexagonal/ pentagonal cells. Errors of (a),(c) height and (b),(d) meridional velocity after 5 days. (c),(d) With or (a),(b) without the hyperviscosity. Note that the color scales are different between (a) and (c), and (b) and (d).

the algorithm of Hasegawa and Tanemura (1976); the same algorithm as Du et al. (2003) for a uniform density function. In the test case 2 of Williamson et al. (1992), more detail of which will be explained in section 3 , the model fails to maintain the balance of a zonal geostrophic flow. For the icosahedral grid with 2562 hexagonal/pentagonal cells, a spurious current (Fig. 3b) continuously takes a significant amount of mass away from the cells along the equator (Fig. 3a). An addition of a $\nabla^{8}$ hyperviscosity term can mitigate this spurious outflow of mass (Fig. 3c) as suggested by Ringler and Randall (2002b), whereas a checkerboard pattern still resides in the meridional velocity field (Fig. 3d). Moreover, errors grow steadily in a later period of 365-day simulations performed on the grids with 2562, 10242 and 40962 hexagonal/pentagonal cells (Fig. 4). We will define the error norms, $L_{2}(h)$ and $L_{\infty}(h)$, in section 3 . These results indicate that this ZM-grid model cannot achieve a stable long-term simulation as those demonstrated in Fig. 11 of Tomita et al. (2001) by an A-grid model and Fig. 5 of Ringler et al. (2010) by a C-grid model. Note that the inclusion of a hyperviscosity term does not fix a computational mode problem of the $\mathrm{ZM}$ grid.

Recently, Miura (2017) proposed another hexagonal B-grid staggering, which was named as the synchronized B grid (SB grid). The SB grid uses the same variable arrangement as the $\mathrm{ZM}$ grid, but discrete forms of the nonlinear terms in the velocity equations are different. The nonlinear terms of the SB grid are 


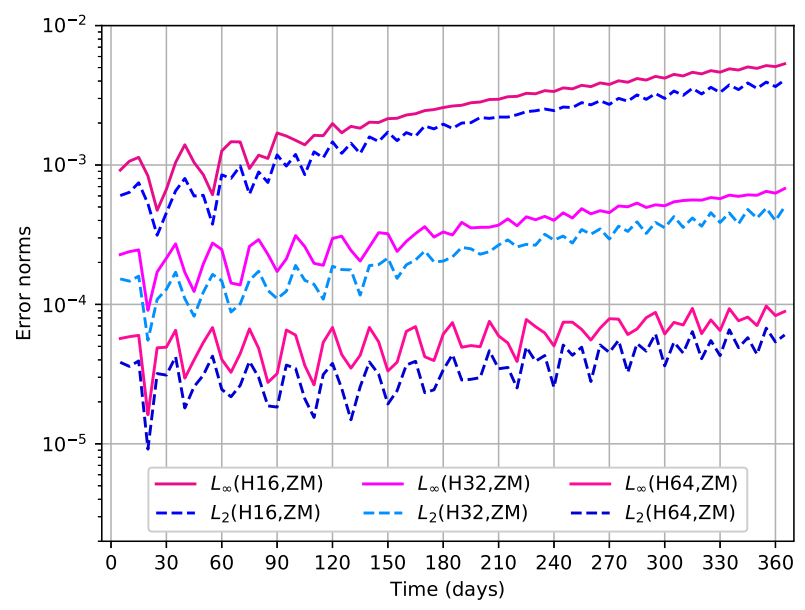

FIG. 4. The test case 2 of Williamson et al. (1992) performed by a ZM-grid model. Time evolutions of $L_{2}(h)$ (solid lines) and $L_{\infty}(h)$ (dashed lines) for the grids with 2562 (H16), 10242 (H32), and 40962 (H64) hexagonal cells.

designed so that the $\mathrm{B} 1$ and $\mathrm{B} 2$ velocity fields (Figs. 2b,c) do not couple each other (Fig. 5b), whereas those of the $\mathrm{ZM}$ grid treat two velocity fields without distinction (Fig. 5a). In the SB-grid system, the complete synchronization of the depth fields of the B1 and B2 systems allows matched evolutions of the B1 and B2 shallow-water systems. Since the differences between the SB-grid and ZM-grid models are only in the discretizations of the nonlinear terms, the dispersion relation of the linearized SB-grid model is as same as the ZM-grid model, and thus also as the Z-grid one (Ringler and Randall 2002b). Miura (2017) demonstrated that the SB-grid model behaved quite similar to the Z-grid model on the planar hexagonal mesh, and the computational mode problem associated with the redundancy of the velocity points was certainly settled.

In this work, we apply the SB-grid system to solve the shallow-water equations on the spherical icosahedral grid. The aim is to examine whether the SB grid is a possible candidate of the horizontal staggering on the spherical icosahedral grid as lower-order finitevolume approach along with the existing A-grid and
C-grid schemes. When we directly followed the approach of Miura (2017), two problems happened: (i) smaller-scale noises appeared in lower latitudes, and (ii) the accuracy of the geostrophic balance was not sufficient. To solve those problems, we revise the original algorithm to include a hyperviscosity term that works to synchronize the velocity fields (Fig. 5c), and also improve the accuracy of the discrete gradient operator.

The rest of this work is organized as follows. In section 2, the SB-grid shallow-water system is introduced first, and then, its application to the spherical icosahedral grid is explained. It contains the derivations of the discrete forms of operators and the way to handle pentagonal cells. Section 3 provides results of some standard test cases to verify that the SB-grid model is almost second-order accurate if flow fields and the bottom surface are smooth. Section 4 is a summary of this study. A quadratic reconstruction of scalar fields inside hexagonal/pentagonal cells is somewhat complicated and is located in appendix A.

\section{The SB-grid shallow-water model}

\section{a. The SB-grid model}

At the beginning, we concisely summarize the SB-grid shallow-water model on a regular hexagonal mesh (Miura 2017). The model uses the vector-invariant formulation of the shallow-water equations:

$$
\begin{aligned}
& \frac{\partial h}{\partial t}=-\nabla \cdot(h \mathbf{v}) \quad \text { and } \\
& \frac{\partial \mathbf{v}}{\partial t}=-(f+\zeta) \mathbf{k} \times \mathbf{v}-\nabla K-\nabla g\left(h+h_{b}\right),
\end{aligned}
$$

where $h$ is fluid depth, $h_{b}$ is the bottom boundary height, $\mathbf{v}$ is horizontal velocity, $\nabla$ is the horizontal gradient operator, $\mathbf{k}$ is the vertical unit vector, $f$ is the Coriolis parameter, $\zeta=\mathbf{k} \cdot \nabla \times \mathbf{v}$ is the vertical component of relative vorticity, $K=\mathbf{v} \cdot \mathbf{v} / 2$ is kinetic energy, and $g$ is the gravitational constant.

In this subsection, variables are denoted with the subscripts 1 and 2 to distinguish between the B1 and the (a) ZM system

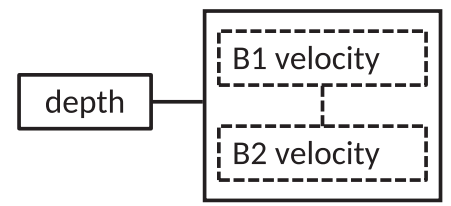

(b) SB system (Miura 2017)

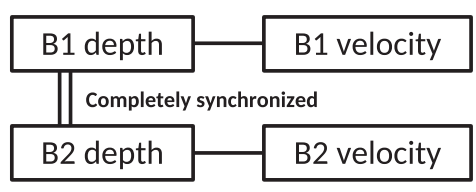

(c) Revised SB system

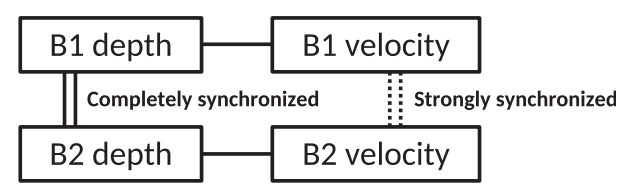

FIG. 5. Schematic illustrations of the relationships between fluid depth and B1 and B2 velocities. (a) The ZM system, (b) the original SB system, and (c) the revised SB system of this work. 

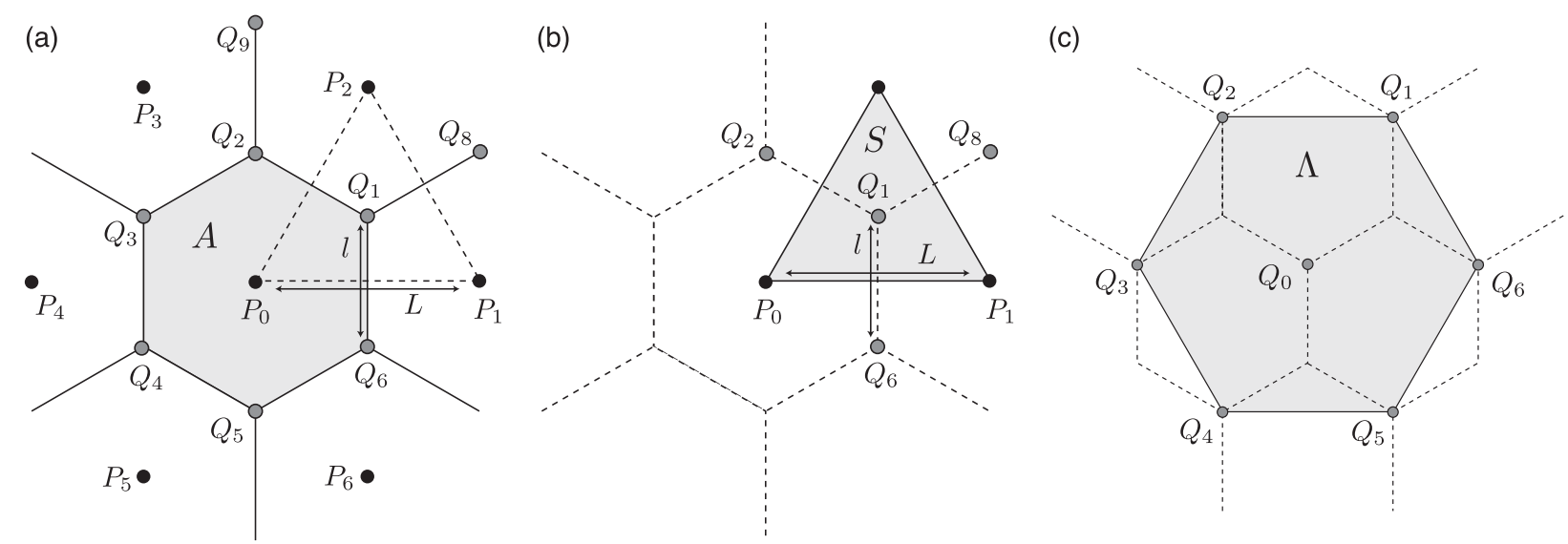

FIG. 6. Schematic illustrations of a regular hexagonal/triangular cell. (a) A hexagonal cell with six cell corners and six surrounding cell centers, (b) a triangular cell, and (c) a large hexagonal cell comprising six hexagonal cell corners.

B2 systems. The B1 grid places $h_{1}$ at the centers of hexagonal cells and $\mathbf{v}_{1}$ at every other vertex of hexagonal cells (Fig. 2b). The B2 grid places $h_{2}$ at the cell centers commonly but $\mathbf{v}_{2}$ at the vertices where $\mathbf{v}_{1}$ is not assigned (Fig. 2c). The prognostic equations of the B1 and $\mathrm{B} 2$ systems are

$$
\begin{aligned}
\left.\frac{\partial h_{1}}{\partial t}\right|_{P_{0}}= & -\left[\nabla_{1} \cdot\left(h_{1} \mathbf{v}_{1}\right)\right]_{P_{0}}-\frac{h_{1, P_{0}}-h_{2, P_{0}}}{2 \tau_{h}}, \\
\left.\frac{\partial h_{2}}{\partial t}\right|_{P_{0}}= & -\left[\nabla_{2} \cdot\left(h_{2} \mathbf{v}_{2}\right)\right]_{P_{0}}-\frac{h_{2, P_{0}}-h_{1, P_{0}}}{2 \tau_{h}}, \\
\left.\frac{\partial \mathbf{v}_{1}}{\partial t}\right|_{Q_{1}}= & -\left(f+\mathbf{k} \cdot \nabla^{*} \times \mathbf{v}_{1}\right)_{Q_{1}}\left(\mathbf{k} \times \mathbf{v}_{1}\right)_{Q_{1}}-\left[\nabla^{*} K_{1}\right]_{Q_{1}}, \\
& -\left[\nabla g\left(h_{1}+h_{b}\right)\right]_{Q_{1}}-\frac{\mathbf{v}_{1, Q_{1}}-\tilde{\mathbf{v}}_{2, Q_{1}}}{2 \tau_{v}} \\
\left.\frac{\partial \mathbf{v}_{2}}{\partial t}\right|_{Q_{2}}= & -\left(f+\mathbf{k} \cdot \nabla^{*} \times \mathbf{v}_{2}\right)_{Q_{2}}\left(\mathbf{k} \times \mathbf{v}_{2}\right)_{Q_{2}}-\left[\nabla^{*} K_{2}\right]_{Q_{2}} \\
& -\left[\nabla g\left(h_{2}+h_{b}\right)\right]_{Q_{2}}-\frac{\mathbf{v}_{2, Q_{2}}-\tilde{\mathbf{v}}_{1, Q_{2}}}{2 \tau_{v}} .
\end{aligned}
$$

The terms with the subscripts $P_{0}, Q_{1}$, or $Q_{2}$ mean that they are evaluated at the cell center or at the cell corners (Fig. 6a), and $\nabla_{s}$ is the discrete nabla operator for the flux divergence of the $\mathrm{B} s$ grid $(s=1,2)$. We will define the specific forms of the discrete flux-divergence, curl, and gradient operators for an irregular hexagonal/ pentagonal mesh later. Note that the gradient operator with no asterisk is defined for a triangular cell configured by three nearest cell centers (Fig. 6b), whereas the curl and gradient operators with one asterisk is defined for a larger hexagonal cell that is made by six cell corners that are two points away from the target corner (Fig. 6c). This definition ensures that the $\mathbf{v}_{1}$ and $\mathbf{v}_{2}$ fields do not interact with each other through the nonlinear terms.

The rightmost terms of (3)-(6) are added to synchronize the B1 and the B2 systems, and $\tau_{h}, \tau_{v}>0$ control the strength of the synchronizations. These formulations are categorized as a bidirectional coupling of two chaotic systems (Fujisaka and Yamada 1983; Boccaletti et al. 2002). For $\tau_{h} \rightarrow 0$, (3) and (4) are transformed to

$$
\begin{aligned}
h_{1} & =h_{2}, \quad \text { and } \\
\left.\frac{\partial h}{\partial t}\right|_{P_{0}} & =-\frac{1}{2}\left[\nabla_{1} \cdot\left(h \mathbf{v}_{1}\right)+\nabla_{2} \cdot\left(h \mathbf{v}_{2}\right)\right]_{P_{0}},
\end{aligned}
$$

where $h=\left(h_{1}+h_{2}\right) / 2$ (Miura 2017).

To synchronize the velocity fields, we need to define $\tilde{\mathbf{v}}_{2, Q_{1}}$ and $\tilde{\mathbf{v}}_{1, Q_{2}}$. Those variables reside at $Q_{1}$ and $Q_{2}$ but belong to the $\mathrm{B} 2$ and the $\mathrm{B} 1$ systems, respectively. Miura (2017) simply assumed $\tilde{\mathbf{v}}_{2, Q_{1}}=\mathbf{v}_{1, Q_{1}}$ and $\tilde{\mathbf{v}}_{1, Q_{2}}=\mathbf{v}_{2, Q_{2}}$ to eliminate the synchronization terms (Fig. 5b). With this definition, however, the coupling of the B1 and B2 systems are only through the fluid depth equations. As a result, the $\mathbf{v}_{1}$ and $\mathbf{v}_{2}$ fields can evolve independently if fluid motions are not associated with height variations, and discrepancies between the B1 and B2 velocities cause small-scale noises as we will see in the next section. To solve this problem, we take another approach to approximate $\tilde{\mathbf{v}}_{2, Q_{1}}$ and $\tilde{\mathbf{v}}_{1, Q_{2}}$ as

$$
\begin{aligned}
& \tilde{\mathbf{v}}_{2, Q_{1}}=\frac{\mathbf{v}_{2, Q_{2}}+\mathbf{v}_{2, Q_{6}}+\mathbf{v}_{2, Q_{8}}}{3}, \text { and } \\
& \tilde{\mathbf{v}}_{1, Q_{2}}=\frac{\mathbf{v}_{1, Q_{1}}+\mathbf{v}_{1, Q_{3}}+\mathbf{v}_{1, Q_{9}}}{3},
\end{aligned}
$$


in effect interpolating $\mathbf{v}_{2}$ to $Q_{1}$ and vice versa. Note that this interpolant is second-order accurate only on uniform hexagonal meshes but first-order accurate if any mesh deformation is present.

If we apply a backward time differencing scheme for the synchronization terms of (5) and (6), the two velocity fields can be synchronized almost completely since $\tau_{v}$ can be tiny enough to be regarded as infinitesimal practically without limitation of the stability. However, this procedure has a concern about its application to massive parallel computers. An inverse problem of a large sparse matrix needs to be solved at every time step. To avoid this computational difficulty, we use a forward time differencing scheme at the expense of the complete synchronization of the velocity fields. As described below, we can use a (hyper)viscosity as a substitute for a synchronization term, and a combination with a proper time discretization allows stable forward-in-time integrations. Note that, in compensation for its suitability for parallel computations, a (hyper)viscosity may pose a stronger constraint on time step size and produce more diffusive solution although these problems did not become evident in the test cases shown in section 3.

Here, we confirm that a viscosity term can be regarded as a synchronization term. On one hand, substituting (9) into the rightmost term of (5), we obtain

$$
\begin{aligned}
-\frac{\mathbf{v}_{1, Q_{1}}-\tilde{\mathbf{v}}_{2, Q_{1}}}{2 \tau_{v}}= & \frac{1}{6 \tau_{v}}\left[\left(\mathbf{v}_{2, Q_{2}}-\mathbf{v}_{1, Q_{1}}\right)+\left(\mathbf{v}_{2, Q_{6}}-\mathbf{v}_{1, Q_{1}}\right)\right. \\
& \left.+\left(\mathbf{v}_{2, Q_{8}}-\mathbf{v}_{1, Q_{1}}\right)\right] .
\end{aligned}
$$

On the other hand, the Laplacian of a scalar $\phi$ may be discretized as

$$
\left.\nabla^{2} \phi\right|_{Q_{1}} \approx \frac{L}{S l}\left[\left(\phi_{Q_{2}}-\phi_{Q_{1}}\right)+\left(\phi_{Q_{6}}-\phi_{Q_{1}}\right)+\left(\phi_{Q_{8}}-\phi_{Q_{1}}\right)\right]
$$

for the regular triangle cell around $Q_{1}$, following Masuda and Ohnishi (1986) and Heikes and Randall (1995) (Fig. 6b). Here, $S$ is the triangle area, $L$ is the distance between the neighboring centers of hexagons, and $l$ is the edge length of the hexagonal cells. Comparing (11) and (12), we find that a viscosity term is equivalent to the synchronization term as

$$
-\frac{\mathbf{v}_{1, Q_{1}}-\tilde{\mathbf{v}}_{2, Q_{1}}}{2 \tau_{v}}=\nu_{2} \nabla^{2} \mathbf{v}
$$

where $\nu_{2}=S l / 6 L \tau_{v}$ is the viscosity coefficient. Since solutions are highly diffusive when (13) is used, we alternatively adopt the hyperviscosity $(-1)^{n-1} \nu_{2 n} \nabla^{2 n} \mathbf{v}$

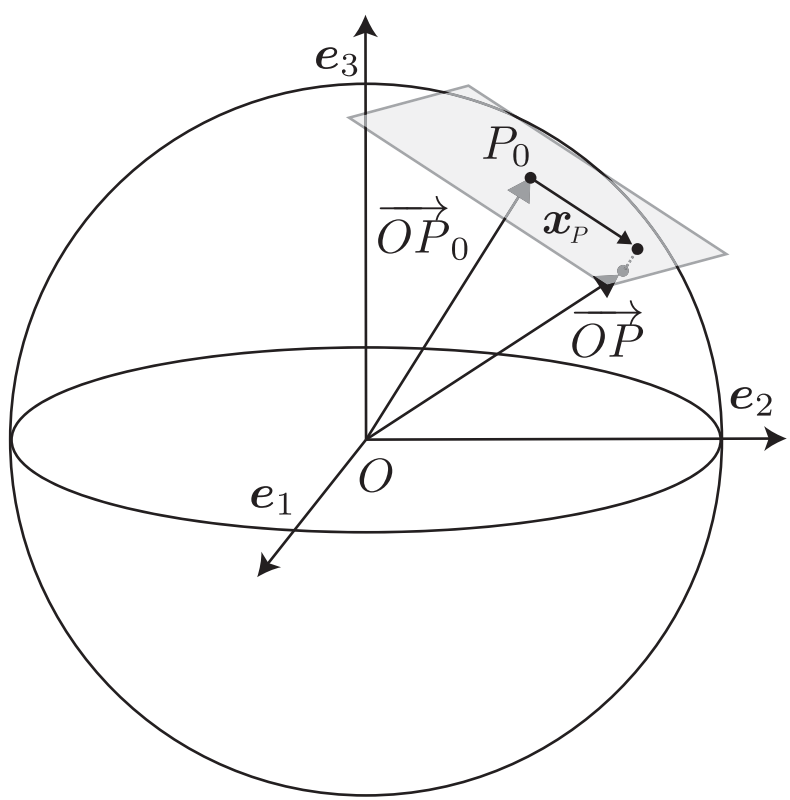

FIG. 7. A unit sphere and the tangential plane at $P_{0}$.

$(n=2,3, \ldots)$ for the synchronization. Finally, the velocity equation for a cell corner $Q_{s}$, which belongs to the $\mathrm{B} s$ system $(s=1,2)$, is given by

$$
\begin{aligned}
\left.\frac{\partial \mathbf{v}_{s}}{\partial t}\right|_{Q_{s}}= & -\left(f+\mathbf{k} \cdot \nabla^{*} \times \mathbf{v}_{s}\right)_{Q_{s}}\left(\mathbf{k} \times \mathbf{v}_{s}\right)_{Q_{s}}-\left(\nabla^{*} K_{s}\right)_{Q_{s}} \\
& -\left[\nabla g\left(h+h_{b}\right)\right]_{Q_{s}}+\left[(-1)^{n-1} \nu_{2 n} \nabla^{2 n} \mathbf{v}\right]_{Q_{s}} .
\end{aligned}
$$

In summary, the SB-grid model comprises (8) and (14). Although the prognostic equations of $h_{1}$ and $h_{2}$ are degenerated, the prognostic variables $h, \mathbf{v}_{1}$ and $\mathbf{v}_{2}$ must evolve under the constraint (7). Note that $\mathbf{k}$. $\nabla^{*} \times \mathbf{v}_{s}$ and $\nabla^{*} K_{s}$ are discretized for a larger hexagonal cell (Fig. 6c), whereas $\nabla g\left(h+h_{b}\right)$ and $\nabla^{2} \mathbf{v}$ are discretized for a smaller triangular cell (Fig. 6b). If we choose a sufficiently large number of $\nu_{2 n}$, the $\mathbf{v}_{1}$ and $\mathbf{v}_{2}$ fields are not completely but strongly synchronized (Fig. 5c).

\section{b. The icosahedral hexagonal/pentagonal mesh}

We choose the origin of a three-dimensional coordinate at the center of a unit sphere (Fig. 7). A complete orthonormal basis of this three-dimensional space is $\mathbf{e}_{i}(i=1,2,3)$. The notation $\overrightarrow{O P}$ indicates the vector pointing from the origin to a point $P$ on the sphere. We can span the plane surface that is tangential to the sphere at a point $P_{0}$ by choosing a set of orthonormal basis $\mathbf{i}, \mathbf{j}$ that satisfies $\mathbf{i} \cdot \mathbf{j}=0, \mathbf{j} \cdot \mathbf{k}=0$ and $\mathbf{k} \cdot \mathbf{i}=0$, where $\mathbf{k}=\overrightarrow{O P_{0}}$. A point $P$ that is in the neighbor of $P_{0}$ and also on the unit sphere can be projected onto this tangential 

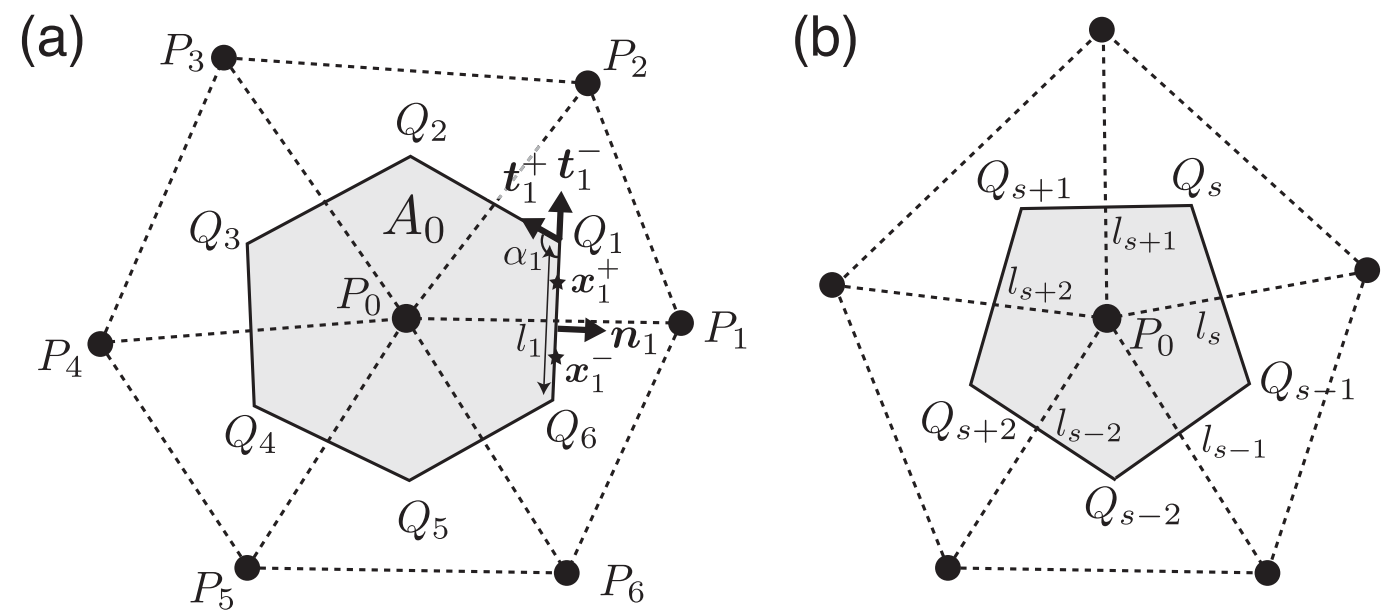

FIG. 8. Schematic illustrations of (a) a hexagonal cell and (b) a pentagonal cell.

plane. We compute the coordinate of the projected point on the tangential plane as

$\mathbf{x}_{P}=\left(x_{P}, y_{P}\right)=\left[\mathbf{i} \cdot\left(\overrightarrow{O P}-\overrightarrow{O P_{0}}\right), \mathbf{j} \cdot\left(\overrightarrow{O P}-\overrightarrow{O P_{0}}\right)\right]$

A spherical icosahedral grid can be constructed from an icosahedron inscribed inside the unit sphere (Masuda and Ohnishi 1986; Heikes and Randall 1995). First, each triangular face is subdivided into four new triangles by bisecting the edges. Next, the new vertices are projected onto the sphere from the origin to create a new polyhedron. Sequentially applying this two-step procedure of subdivision and projection, we obtain a triangular mesh of an expected resolution. We indicate the nodes of a triangular mesh by $P_{i}(i=0,1,2, \ldots)$. All neighboring nodes are connected by great circles to configure a spherical triangular mesh. When not mentioned explicitly, the locations of the node points are optimized by the algorithm of Hasegawa and Tanemura (1976), which is equivalent to the algorithm of Du et al. (2003) of a uniform density function.

We denote the barycenter of a spherical triangle $P_{0} P_{i} P_{i+1}$ as $Q_{i}$ (Fig. 8), the location of which is computed by

$$
\overrightarrow{O Q_{i}}=\frac{\overrightarrow{O P_{0}}+\overrightarrow{O P_{i}}+\overrightarrow{O P_{i+1}}}{\left|\overrightarrow{O P_{0}}+\overrightarrow{O P}_{i}+\overrightarrow{O P_{i+1}}\right|}
$$

We configure a spherical hexagonal/pentagonal mesh of the barycentric type (Fig. 1b) by connecting all neighboring barycenters by great circles. The vertices $Q_{i}(i=$ $1,2, \ldots, N)$, where $N$ is the number of the vertices $(N=6$ for hexagons and $N=5$ for pentagons), locate counterclockwise, viewed from the outside of the unit sphere.
The reason why we adopt the barycentric type is that the cell averaging over the triangle $P_{0} P_{i} P_{i+1}$ is second-order accurate at the point $Q_{i}$ and the accuracy degrades elesewhere. The total number of hexagonal/pentagonal cells after $m$ th recursive subdivisions/projections are $10 \times 2^{2 m}+2$, including 12 pentagons locating at the 12 vertices of the original icosahedron. Tomita et al. (2001) introduced a notation "glevel- $m$ " to mention it.

The unit vector normal to the circular sector $O Q_{i-1} Q_{i}$ and directed outward from the $P_{0}$ cell is $\mathbf{n}_{i}=\overrightarrow{O Q_{i}} \times$ $\overrightarrow{O Q_{i-1}}$. The notation $i$ is cyclic, that is, $i-1$ means $1+$ modulo $(i-2, N)$ and $i+1$ does $1+\bmod (i, N)$, if we use the standard intrinsic procedures of FORTRAN 90. The unit vector tangential to the arc $\widehat{Q}_{i-1} Q_{i}$ and directed from $Q_{i-1}$ to $Q_{i}$ at $Q_{i}$ is $\mathbf{t}_{i}^{-}=\overrightarrow{O Q_{i}} \times \mathbf{n}_{i}$. Similarly, the tangential unit vector along the arc $Q_{i} Q_{i+1}$ directing from $Q_{i}$ to $Q_{i+1}$ at $Q_{i}$ is $\mathbf{t}_{i}^{+}=\overrightarrow{O Q_{i}} \times \mathbf{n}_{i+1}$. Thus, a set of orthonormal basis at $Q_{i}$ can be $(\mathbf{i}, \mathbf{j})_{Q_{i}}=\left(\mathbf{n}_{i}, \mathbf{t}_{i}^{-}\right)$or $(\mathbf{i}, \mathbf{j})_{Q_{i}}=\left(\mathbf{n}_{i+1}, \mathbf{t}_{i}^{+}\right)$. Another choice may be the pair of the eastward and northward unit vectors at $Q_{i}$. The interior angle of the $P_{0}$ cell at the vertex $Q_{i}$ can be computed by $\alpha_{i}=$ $\cos ^{-1}\left(-\mathbf{t}_{i}^{-} \cdot \mathbf{t}_{i}^{+}\right)$, but $\alpha_{i}=\tan ^{-1}\left[\left|-\mathbf{t}_{i}^{-} \times \mathbf{t}_{i}^{+}\right| /\left(\mathbf{t}_{i}^{-} \cdot \mathbf{t}_{i}^{+}\right)\right]$may be a better choice for computational accuracy.

The length of a great circle $\overparen{P Q}$ is $l=\tan ^{-1}[\mid \overrightarrow{O P} \times$ $\overrightarrow{O Q} \mid / \overrightarrow{O P} \cdot \overrightarrow{O Q}]$. Given three interior angles $\alpha_{i}(i=1,2$, 3 ) at the vertices $P_{i}$, the area of a spherical triangle $P_{1} P_{2}$ $P_{3}$ is $S=\alpha_{1}+\alpha_{2}+\alpha_{3}-\pi$. Although it was not the case in this work, this Girard's formula may not be accurate enough in high resolutions. If it happens, we might try other choices such as L'Huilier's formula.

\section{c. The flux-divergence operator}

We denote the area integral of a scalar $q$ over the hexagonal/pentagonal cell that contains the node point $P_{i}$ as 


$$
\langle q\rangle_{i}=\int_{A_{i}} q(\mathbf{x}) d A
$$

where $A_{i}$ is the area of the $P_{i}$ cell. Inversely, given $\langle q\rangle_{i}$ for all hexagonal/pentagonal cells, we want to approximate the distribution $q(\mathbf{x})$ in the neighbor of $P_{i}$ by a quadratic reconstruction $\tilde{q}_{i}(\mathbf{x})$. Appendix A summarizes the method of a quadratic reconstruction that satisfies

$$
\langle q\rangle_{0}=\int_{A_{0}} \tilde{q}_{0}(\mathbf{x}) d A
$$

for the cell of interest, and approximates

$$
\langle q\rangle_{i}=\int_{A_{i}} \tilde{q}_{0}(\mathbf{x}) d A \quad(i=1, \ldots, N)
$$

for the surrounding cells $(N=6$ for hexagons and $N=5$ for pentagons).
For a hexagonal cell that contains $P_{0}$, we integrate (8) over the cell area to obtain

A unique treatment is needed for the pentagons, and we will describe it later. Instead of $h$, we adopt fluid mass $\langle h\rangle$ as a prognostic variable. The value of $h$ at a point $P$ about $P_{0}$ can be approximated as $\tilde{h}_{P}=\tilde{h}_{0}\left(\mathbf{x}_{P}\right)$ to the third-order accuracy. The usage of the area averaging $\bar{h}_{0}=\langle h\rangle_{0} / A_{0}$ is limited to the output and to evaluations of conservations. This area averaging is second-order accurate at most only at the barycenter of the $P_{0}$ cell, but does not influence simulation results since our model does not use $\bar{h}_{0}$ in time integrations.

The right-hand side of (20) is spatially discretized as

$$
\begin{aligned}
\frac{\partial\langle h\rangle_{0}}{\partial t} & =-\frac{1}{2}\left(\sum_{i=1,2} \hat{h}_{i} l_{i} \mathbf{n}_{i} \cdot \mathbf{v}_{1}+\sum_{i=3,4} \hat{h}_{i} l_{i} \mathbf{n}_{i} \cdot \mathbf{v}_{3}+\sum_{i=5,6} \hat{h}_{i} l_{i} \mathbf{n}_{i} \cdot \mathbf{v}_{5}\right)_{1}-\frac{1}{2}\left(\sum_{i=2,3} \hat{h}_{i} l_{i} \mathbf{n}_{i} \cdot \mathbf{v}_{2}+\sum_{i=4,5} \hat{h}_{i} l_{i} \mathbf{n}_{i} \cdot \mathbf{v}_{4}+\sum_{i=6,1} \hat{h}_{i} l_{i} \mathbf{n}_{i} \cdot \mathbf{v}_{6}\right)_{2} \\
& =-\frac{1}{2} \sum_{i=1}^{6} \hat{h}_{i} l_{i}\left(\mathbf{n}_{i} \cdot \mathbf{v}_{i}+\mathbf{n}_{i} \cdot \mathbf{v}_{i-1}\right),
\end{aligned}
$$

where $\hat{h_{i}}$ is an estimate of the fluid depth for the $i$ th edge, $l_{i}$ is the length of the $i$ th edge, and $\mathbf{v}_{i}$ is the velocity vector at $Q_{i}$ (Fig. 8a).

Following Miura (2013), $\hat{h}_{i}$ is determined by using the quadratic profiles $\tilde{h}_{0}(\mathbf{x})$ and $\tilde{h}_{i}(\mathbf{x})$. The line integral of those quadratic profiles can be computed by using two approximate quadrature points

$$
\overrightarrow{O G_{i}^{ \pm}}=\frac{(1 \mp 1 / \sqrt{3}) \overrightarrow{O Q_{i-1}}+(1 \pm 1 / \sqrt{3}) \overrightarrow{O Q_{i}}}{\left|(1 \mp 1 / \sqrt{3}) \overrightarrow{O Q_{i-1}}+(1 \pm 1 / \sqrt{3}) \overrightarrow{O Q_{i}}\right|}
$$

as

$$
\begin{aligned}
& \hat{h}_{i}^{L} l_{i}=\int_{l_{i}} \tilde{h}_{0}(\mathbf{x}) d l=\frac{\tilde{h}_{0}\left(\mathbf{x}_{i}^{+}\right)+\tilde{h}_{0}\left(\mathbf{x}_{i}^{-}\right)}{2} l_{i}, \quad \text { and } \\
& \hat{h}_{i}^{R} l_{i}=\int_{l_{i}} \tilde{h}_{i}(\mathbf{x}) d l=\frac{\tilde{h}_{i}\left(\mathbf{x}_{i}^{+}\right)+\tilde{h}_{i}\left(\mathbf{x}_{i}^{-}\right)}{2} l_{i} .
\end{aligned}
$$

Note that we may divide the geodesic arc $\widehat{Q}_{i-1} Q_{i}$ to obtain the quadrature points as an alternative to (22). The coordinate of $G_{i}^{ \pm}$, denoted by $\mathbf{x}_{i}^{ \pm}$, are computed by (15) about $P_{0}$ for (23) and about $P_{i}$ for (24). Similar to Skamarock and Gassmann (2011) and Miura (2013),

$$
\begin{aligned}
& \hat{h}_{i}=\gamma \hat{h}_{i}^{L}+(1-\gamma) \hat{h}_{i}^{R}, \\
& \gamma=\left[1+\beta \operatorname{sign}\left(\mathbf{n}_{i} \cdot \mathbf{v}_{i}+\mathbf{n}_{i} \cdot \mathbf{v}_{i-1}\right)\right] / 2,
\end{aligned}
$$

where

$$
\operatorname{sign}(v) \equiv\left\{\begin{array}{c}
-1 \text { for } v<0 \\
1 \text { for } v \geq 0
\end{array} .\right.
$$

We use $\beta=0$ in this work to avoid implicit diffusions of an upwinding. In practical applications, we may use $0<\beta \leq 1$ to receive benefits of an upwinding if the same scheme is used for scalar transports. Although it is not used in this work, a flux limiter of Miura (2013), which slightly improves Thuburn $(1995,1996)$, may be combined for the positive definiteness.

This flux-divergence operator behaves similar to the scheme proposed by Miura (2013). This seems a natural consequence from the fact that the two schemes determine the same interface values if they are applied in a one-dimensional equally spaced grid. At pentagonal cells (Fig. 8b), five different systems overlap, as two systems do at hexagons. Appendix B describes a method to synchronize those five systems, and as a result, (21) is slightly modified to be 
(a)

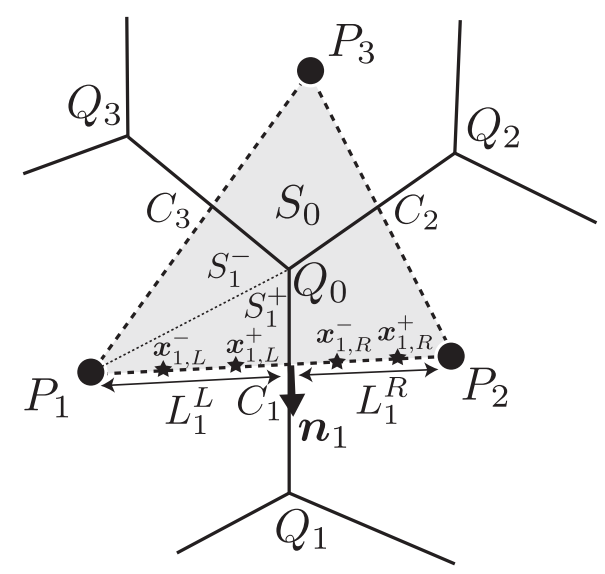

(b)

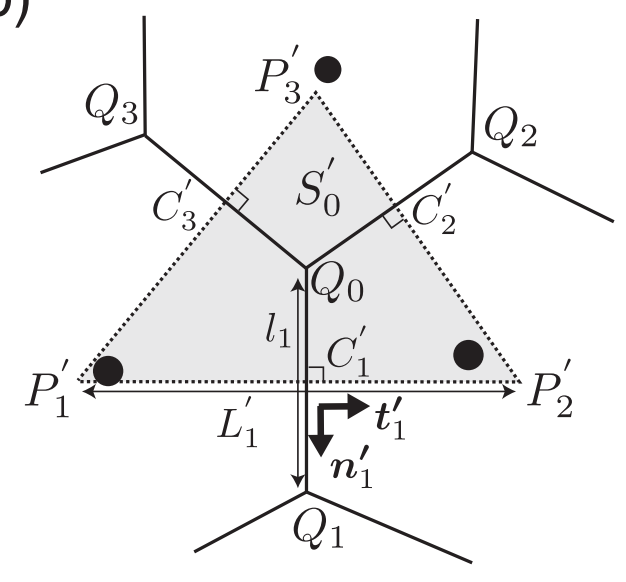

FIG. 9. Schematic illustrations of (a) a triangular cell for the gradient without asterisk and (b) a triangular cell specifically composed for the hyperviscosity.

$$
\frac{\partial\langle h\rangle_{0}}{\partial t}=-\frac{1}{2} \sum_{i=1}^{5} \hat{h}_{i} l_{i}\left(\mathbf{n}_{i} \cdot \mathbf{v}_{i}+\mathbf{n}_{i} \cdot \mathbf{v}_{i-1}\right)
$$

\section{d. The gradient operator for the linear term}

We define a form of discrete gradient operator at cell vertices $Q_{i}$ to evaluate $\nabla g\left(h+h_{b}\right)=g \nabla h_{a}$ in (14), where $h_{a}=h+h_{b}$. Assume that a cell vertex $Q_{0}$ is surrounded by three nodes $P_{i}$ and three vertices $Q_{i}(i=1,2,3)$ (Fig. 9a). We compute the area integrals of the fluid surface height as $\left\langle h_{a}\right\rangle_{i}=\langle h\rangle_{i}+\bar{h}_{b, i} A_{i}$, where $\bar{h}_{b, i}$ is the mean height of the bottom boundary of the $i$ th cell. Given $\left\langle h_{a}\right\rangle$ of all hexagonal/pentagonal cells, we reconstruct quadratic profiles $\tilde{h}_{a, i}(\mathbf{x})$ about $P_{i}$ by the method of appendix A. The arc $\widehat{P_{i} P_{i+1}}$ is partitioned into two segments, $\overparen{P_{i} C_{i}}$ and $\widehat{C_{i} P_{i+1}}$, by the intersection point of $\widehat{P_{i} P_{i+1}}$ and $\widehat{Q_{0} Q_{i}}$. The location of the intersection point is

$$
\overrightarrow{O C}_{i}=\frac{\left(\overrightarrow{O P_{i+1}} \times \overrightarrow{O P_{i}}\right) \times\left(\overrightarrow{O Q_{0}} \times \overrightarrow{O Q_{i}}\right)}{\left|\left(\overrightarrow{O P_{i+1}} \times \overrightarrow{O P_{i}}\right) \times\left(\overrightarrow{O Q_{0}} \times \overrightarrow{O Q_{i}}\right)\right|}
$$

We use two approximate quadrature points for each segment as

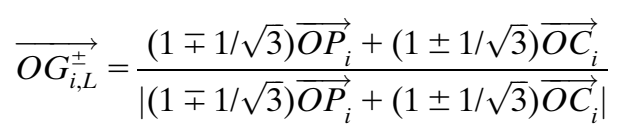

for $\overparen{P_{i} C_{i}}$ and

$$
\overrightarrow{O G_{i, R}^{ \pm}}=\frac{(1 \mp 1 / \sqrt{3}) \overrightarrow{O C}_{i}+(1 \pm 1 / \sqrt{3}) \overrightarrow{O P_{i+1}}}{\left|(1 \mp 1 / \sqrt{3}) \overrightarrow{O C}_{i}+(1 \pm 1 / \sqrt{3}) \overrightarrow{O P_{i+1}}\right|}
$$

for $\overline{C_{i} P_{i+1}}$ to compute line integrals of the quadratic profiles.

We use a relation of the vector calculus, $\mathbf{u} \cdot \nabla h_{a}=$ $\nabla \cdot\left(h_{a} \mathbf{u}\right)-h_{a} \nabla \cdot \mathbf{u}$, with a spatially uniform vector $\mathbf{u}$. The second term on the right-hand side is retained since the area integral of $\nabla \cdot \mathbf{u}$ does not necessarily vanish in a discrete form. Taking the area integral over the spherical triangle $P_{1} P_{2} P_{3}$, the area of which is $S_{0}$, the righthand side can be rewritten in the form of line integrals as

$$
\begin{aligned}
\mathbf{u} \cdot \int_{S_{0}} \nabla h_{a} d S & =\mathbf{u} \cdot \int_{L} h_{a} \mathbf{n} d L-\int_{S_{0}} h_{a} \nabla \cdot \mathbf{u} d S \\
& \approx \mathbf{u} \cdot \int_{L}\left(h_{a}-\bar{h}_{a}\right) \mathbf{n} d L
\end{aligned}
$$

where we assume that $h_{a}$ inside the integral in the second term on the right-hand side is represented by a constant $\bar{h}_{a}$.

Using (32), we define the discrete form of gradient for the linear term at $Q_{0}$ as

$$
\begin{aligned}
\left.\nabla h_{a}\right|_{Q_{0}} & =\lim _{S_{0} \rightarrow 0} \frac{1}{S_{0}} \int_{S_{0}} \nabla h_{a} d S \\
& \approx \frac{1}{S_{0}} \sum_{i=1}^{3}\left[\left(\hat{h}_{a, i}^{L}-\bar{h}_{a}\right) L_{i}^{L}+\left(\hat{h}_{a, i}^{R}-\bar{h}_{a}\right) L_{i}^{R}\right] \mathbf{n}_{i},
\end{aligned}
$$

where $L_{i}^{L}$ and $L_{i}^{R}$ are the lengths of the arcs $\overparen{P_{i} C_{i}}$ and $\widehat{C_{i} P_{i+1}}$, respectively; $\mathbf{n}_{i}=\overrightarrow{O P_{i+1}} \times \overrightarrow{O P_{i}}$,

$$
\begin{aligned}
\hat{h}_{a, i}^{L} & =\frac{1}{2}\left[\tilde{h}_{a, i}\left(\mathbf{x}_{i, L}^{+}\right)+\tilde{h}_{a, i}\left(\mathbf{x}_{i, L}^{-}\right)\right], \\
\hat{h}_{a, i}^{R} & =\frac{1}{2}\left[\tilde{h}_{a, i+1}\left(\mathbf{x}_{i, R}^{+}\right)+\tilde{h}_{a, i+1}\left(\mathbf{x}_{i, R}^{-}\right)\right],
\end{aligned}
$$


and $\bar{h}_{a}$ is the mean height for the perimeter of the triangle, which is determined by

$$
\bar{h}_{a}=\sum_{i=1}^{3}\left(\hat{h}_{a, i}^{L} L_{i}^{L}+\hat{h}_{a, i}^{R} L_{i}^{R}\right) / \sum_{i=1}^{3}\left(L_{i}^{L}+L_{i}^{R}\right) .
$$

The coordinate of the quadrature points $G_{i, L}^{ \pm}$and $G_{i, R}^{ \pm}$ are denoted by $\mathbf{x}_{i, L}^{ \pm}$and $\mathbf{x}_{i, R}^{ \pm}$, and they are computed about the node points $P_{i}$ and $P_{i+1}$, respectively. Note that the index $i$ is cyclic. It is necessary to choose the barycentric-type mesh to ensure the second-order accuracy of the area averaging in (33) at the point $Q_{0}$. This discrete form of gradient is a second-order accurate approximation since the line integrals are estimated to the fourth-order accuracy.

\section{e. The Laplacian operator for the hyperviscosity}

A form of discrete Laplacian is defined at cell vertices $Q_{i}$ to compute the hyperviscosity in (14). With a pair of orthonormal basis $(\mathbf{i}, \mathbf{j})$ at a vertex $Q_{0}$, a vector $\mathbf{q}$ at $Q_{0}$ can be expressed in a component form as $\mathbf{q}=q_{i} \mathbf{i}+q_{j} \mathbf{j}$, where $q_{i}=\mathbf{i} \cdot \mathbf{q}$ and $q_{j}=\mathbf{j} \cdot \mathbf{q}$. Using a vector calculus, $\nabla^{2} \mathbf{v}$ can be rewritten as

$$
\nabla^{2} \mathbf{v}=\nabla \delta-\nabla \times \zeta=\nabla \delta-[(\mathbf{j} \cdot \nabla \zeta) \mathbf{i}-(\mathbf{i} \cdot \nabla \zeta) \mathbf{j}],
$$

where $\delta=\nabla \cdot \mathbf{v}$ and $\zeta=0 \mathbf{i}+0 \mathbf{j}+\zeta \mathbf{k}$ with $\mathbf{k}=\overrightarrow{O Q_{0}}$. Thus, we can compute the Laplacian of the components of $\mathbf{v}$ if the methods to compute $\delta, \zeta$ and the gradients of them are determined.

We assume that a cell vertex $Q_{0}$ is inside a triangle $P_{1}^{\prime} P_{2}^{\prime} P_{3}^{\prime}$ and is surrounded by three vertices $Q_{i}(i=1,2,3)$ (Fig. 9b). The location of $P_{i}^{\prime}$ is different from $P_{i}$ in general and is defined as follows. First, the unit vector normal to the circular sector $O Q_{0} Q_{i}$ and directed outward from the $P_{i}$ cell is $\mathbf{t}_{i}^{\prime}=\overrightarrow{O Q_{0}} \times \overrightarrow{O Q_{i}}$. Next, the unit vector that is tangential to the arc $Q_{0} Q_{i}$ at the midpoint $C_{i}^{\prime}$ and is directed from $Q_{0}$ to $Q_{i}$ is $\mathbf{n}_{i}^{\prime}=\mathbf{t}_{i}^{\prime} \times \overrightarrow{O C}_{i}$. Finally, we compute the location of $P_{i}^{\prime}$ as

$$
\overrightarrow{O P_{i}^{\prime}}=\frac{\mathbf{n}_{i-1}^{\prime} \times \mathbf{n}_{i}^{\prime}}{\left|\mathbf{n}_{i-1}^{\prime} \times \mathbf{n}_{i}^{\prime}\right|}
$$

The index $i$ is cyclic. The arc $\widehat{P_{i}^{\prime} P_{i+1}^{\prime}}$ bisects the arc $\widehat{Q_{0} Q_{i}}$ at $C_{i}^{\prime}$, and these arcs are orthogonal to each other. The triangle $P_{1}^{\prime} P_{2}^{\prime} P_{3}^{\prime}$ does not generally match the triangle $P_{1}$ $P_{2} P_{3}$. This extra triangle is required since we want to use $\delta$ and $\zeta$ that are evaluated at $C_{i}^{\prime}$ as

$$
\begin{aligned}
& \tilde{\delta}_{i}=\frac{\mathbf{v}_{0} \cdot\left(\overrightarrow{O Q_{0}} \times \mathbf{t}_{i}^{\prime}\right)-\mathbf{v}_{i} \cdot\left(\overrightarrow{O Q_{i}} \times \mathbf{t}_{i}^{\prime}\right)}{l_{i}} \text { and } \\
& \tilde{\zeta}_{i}=\frac{-\mathbf{v}_{0} \cdot \mathbf{t}_{i}^{\prime}+\mathbf{v}_{i} \cdot \mathbf{t}_{i}^{\prime}}{l_{i}}
\end{aligned}
$$

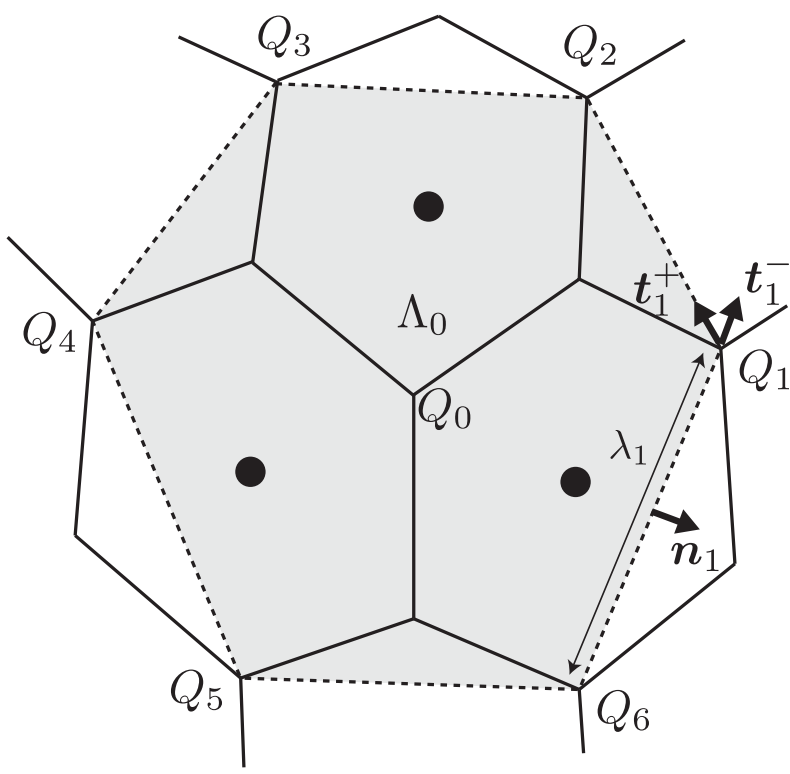

FIG. 10. A schematic illustration of a large hexagonal cell about the vertex $Q_{0}$.

so that the Laplacian is sensitive to deformations of velocity fields at the smallest scale. Where, $\mathbf{v}_{0}$ and $\mathbf{v}_{i}$ are velocity vectors at $Q_{0}$ and $Q_{i}$, respectively, and $l_{i}$ is the length of the arc $\widehat{Q_{0} Q_{i}}$.

The discrete form of gradient in (37) for a scalar $q$ is

$$
\left.\nabla q\right|_{Q_{0}} \approx \frac{1}{S_{0}^{\prime}} \sum_{i=1}^{3} \tilde{q}_{i} L_{i}^{\prime} \mathbf{n}_{i}^{\prime},
$$

where $S_{0}^{\prime}$ is the area of the spherical triangle $P_{1}^{\prime} P_{2}^{\prime} P_{3}^{\prime}, L_{i}^{\prime}$ is the length of the arc $\widehat{P_{i}^{\prime} P_{i+1}^{\prime}}$, and $\tilde{q}_{i}$ is the scalar value evaluated at $C_{i}^{\prime}$.

While (39) and (40) are second-order accurate approximations, the accuracy of the discrete Laplacian is limited to the first order since $C_{i}^{\prime}$, where $\tilde{\delta}_{i}$ and $\tilde{\zeta}_{i}$ are evaluated, is not at the midpoint of the $\operatorname{arc} P_{i} \widehat{P}_{i+1}$, and $Q_{0}$ does not generally coincide with the barycenter of the triangle $P_{1}^{\prime} P_{2}^{\prime} P_{3}^{\prime}$. In addition, we simply ignore the correction term, the second term inside the parentheses of (32), in the derivation of (41); this lower-order formula may be sufficient for the purpose of the synchronization of velocity vectors.

\section{f. The curl and gradient operators for the nonlinear terms}

We configure the curl and gradient operators with one asterisk in (14) using a larger hexagonal cell comprising six cell corners that are two points away from the target point. Assume that the vertex $Q_{0}$ is surrounded by $Q_{i}$ $(i=1, \ldots, 6)$ (Fig. 10). This assumption is valid, even when $Q_{0}$ is a vertex of a pentagonal cell. The area of the 
(a) Z

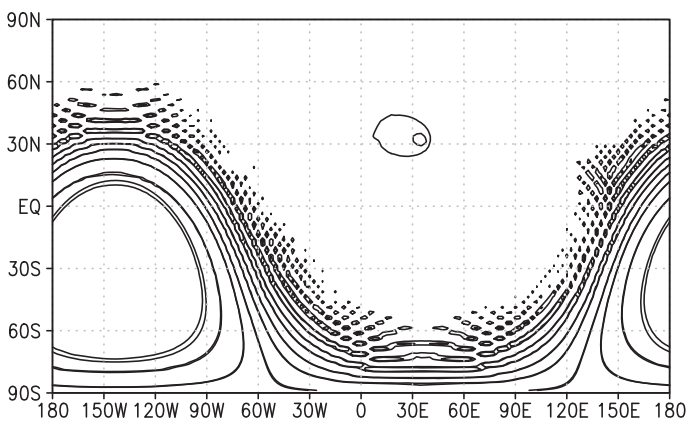

(c) SB

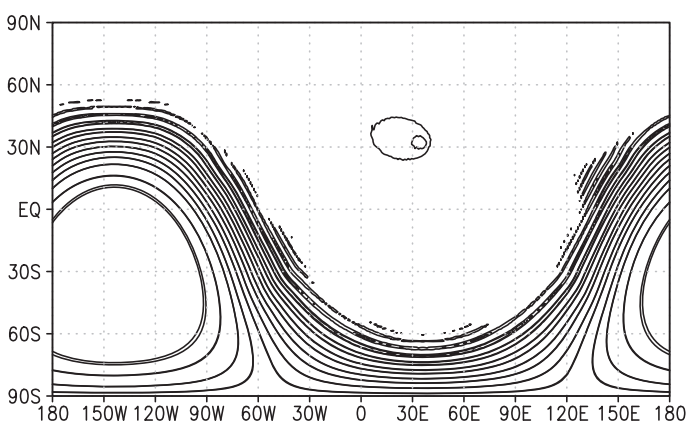

(b) SB (1st-order gradient)

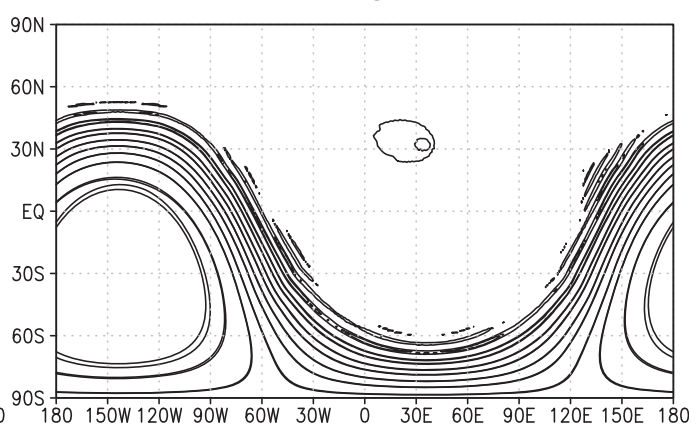

(d) SB $\nu_{8}=0$

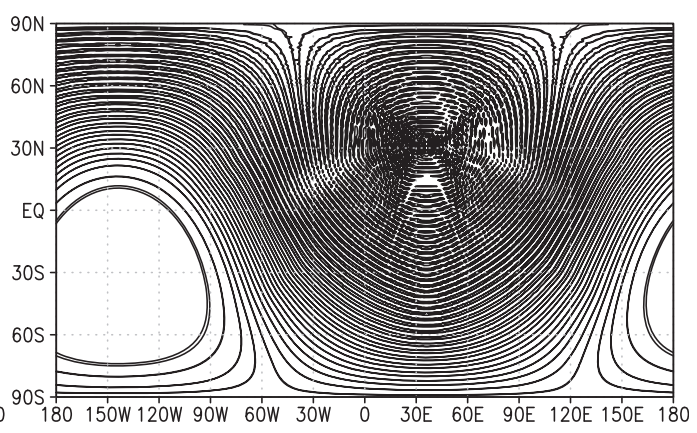

FIG. 11. The one-point forcing test. Height after $24 \mathrm{~h}$ for H64. (a) The Z-grid model and the SB-grid model that used (b) a first-order accurate gradient or (c),(d) the second-order accurate gradient. The SB-grid model was run (b),(c) with or (d) without the hyperviscosity. Contour lines of 2998.2 and $2998.3 \mathrm{~m}$ are drawn.

spherical hexagonal cell is $\Lambda_{0}$, the length of the arc $\vec{Q}_{i-1} Q_{i}$ is $\lambda_{i}, \mathbf{n}_{i}=\overrightarrow{O Q_{i}} \times \overrightarrow{O Q_{i-1}}, \mathbf{t}_{i}^{-}=\overrightarrow{O Q_{i}} \times \mathbf{n}_{i}$, and $\mathbf{t}_{i}^{+}=\overrightarrow{O Q_{i}} \times \mathbf{n}_{i+1}$. The index $i$ is cyclic. Then, the discrete forms of curl and gradient are defined as

$$
\begin{aligned}
\mathbf{k} \cdot \nabla^{*} \times\left.\mathbf{v}\right|_{Q_{0}} & \approx \frac{1}{2 \Lambda_{0}} \sum_{i=1}^{6} \lambda_{i}\left(\mathbf{t}_{i}^{-} \cdot \mathbf{v}_{i}+\mathbf{t}_{i-1}^{+} \cdot \mathbf{v}_{i-1}\right), \text { and } \\
\left.\nabla^{*} K\right|_{Q_{0}} & \approx \frac{1}{2 \Lambda_{0}} \sum_{i=1}^{6}\left(K_{i}+K_{i-1}-2 \bar{K}\right) \lambda_{i} \mathbf{n}_{i},
\end{aligned}
$$

where $\mathbf{v}_{i}$ is velocity vector at $Q_{i}, K_{i}=\mathbf{v}_{i} \cdot \mathbf{v}_{i} / 2$, and

$$
\bar{K}=\sum_{i=1}^{6}\left(K_{i}+K_{i-1}\right) \lambda_{i} / 2 \sum_{i=1}^{6} \lambda_{i}
$$

The accuracy of these discrete forms may be degraded from the second order due to the displacement of $Q_{0}$ from the barycenter of the larger hexagon and also due to distortion of the cell shape. If we want to ensure second-order accuracy, the least squares fitting method of Stuhne and Peltier (1996) and Majewski et al. (2002) can be an option. Here, we prefer (42) and (43) since they have a good property that the area weighted global summation converges to zero as resolution increases.

\section{g. Evaluations of the potential enstrophy and total energy conservations}

We have already determined all discrete forms of spatial derivatives required for time integrations of the SB-grid shallow-water model. Here, we additionally define the methods to evaluate conservations of potential enstrophy and total energy in this work.

First, we follow the notation of Fig. 8, which has been explained in sections $2 \mathrm{~b}$ and $2 \mathrm{c}$. We approximate relative vorticity at $P_{0}$ as

$$
\bar{\zeta}_{0} \approx \frac{1}{A_{0}} \sum_{i=1}^{N} l_{i}\left(\mathbf{t}_{i}^{-} \cdot \mathbf{v}_{i}+\mathbf{t}_{i-1}^{+} \cdot \mathbf{v}_{i-1}\right) .
$$

Given $\bar{h}_{i}=\langle h\rangle_{i} / A_{i}$ and $\bar{\zeta}_{i}(i=0,1,2, \ldots)$, we compute the area-weighted global summation of potential vorticity by

$$
Z=\sum_{i} \frac{\langle h\rangle_{i}}{2}\left(\frac{f_{i}+\bar{\zeta}_{i}}{\bar{h}_{i}}\right)^{2},
$$

where the summation is taken for all hexagonal/pentagonal cells, and $f_{i}$ is the Coriolis parameter at the point $P_{i}$. 


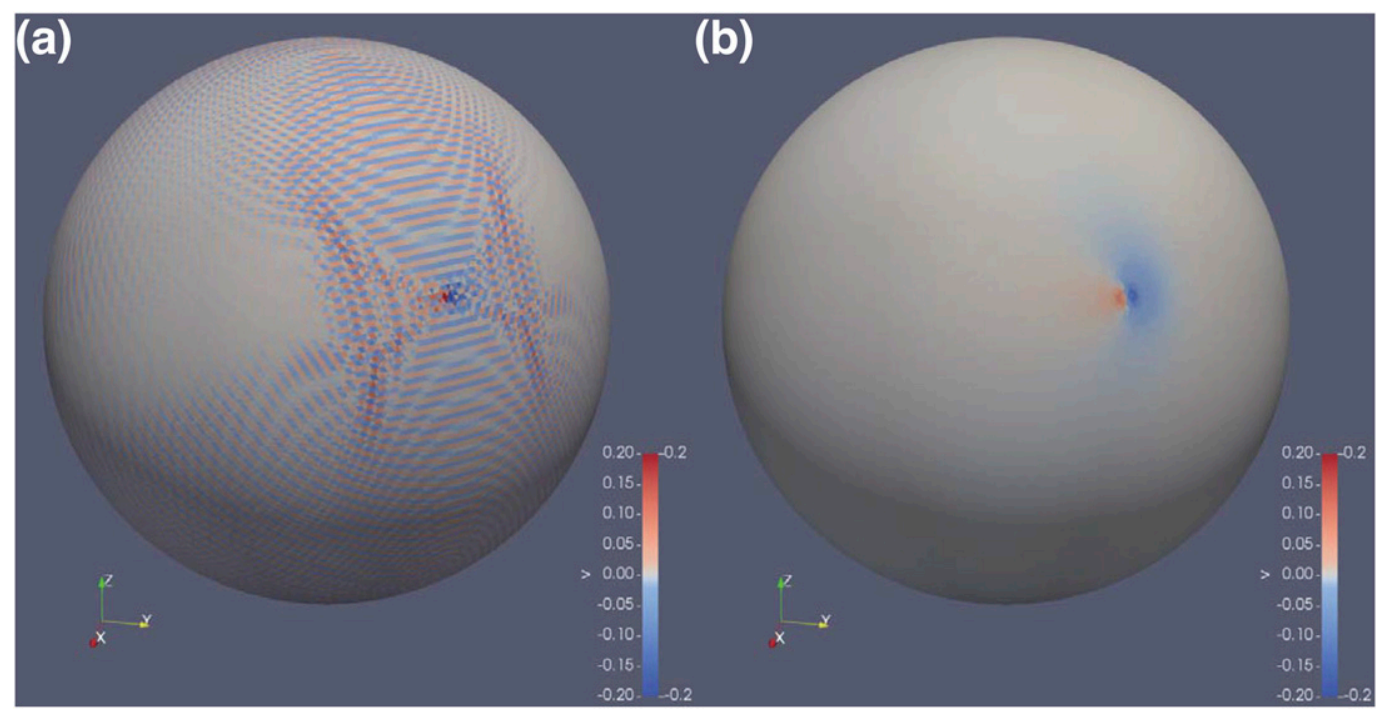

FIG. 12. The one-point forcing test. Meridional velocity after $24 \mathrm{~h}$ for H64 (b) with or (a) without the hyperviscosity.

Next, we follow the notations of Fig. 9a, which has been explained in section 2 d. Here, we need to determine fluid mass of the triangular cell $P_{1} P_{2} P_{3}$, which is denoted by $\langle\tilde{h}\rangle_{0}$. Given a quadratic reconstruction $\tilde{h}_{1}(\mathbf{x})$ about $P_{1}$, the contribution from the $P_{1}$ cell is

$$
\begin{aligned}
\int_{P_{1} C_{1} Q_{0} C_{3}} h d s= & S_{1}^{+} \frac{\tilde{h}_{1}\left(\mathbf{x}_{1}\right)+\tilde{h}_{1}\left(\mathbf{x}_{2}\right)+\tilde{h}_{1}\left(\mathbf{x}_{5}\right)}{3} \\
& +S_{1}^{-} \frac{\tilde{h}_{1}\left(\mathbf{x}_{3}\right)+\tilde{h}_{1}\left(\mathbf{x}_{4}\right)+\tilde{h}_{1}\left(\mathbf{x}_{5}\right)}{3},
\end{aligned}
$$

where $\mathbf{x}_{i}(i=1, \ldots, 5)$ are the coordinates of the midpoints of the arcs $\overparen{P_{1} C_{1}}, \widehat{Q_{0} C_{1}}, \widehat{Q_{0} C_{3}}, \widehat{P_{1} C_{3}}$ and $\widehat{Q_{0} P_{1}}$ about $P_{1}$, respectively, and $S_{1}^{+}$and $S_{1}^{-}$are the areas of the spherical triangles $P_{1} C_{1} Q_{0}$ and $P_{1} Q_{0} C_{3}$, respectively. Accumulating the contributions from $P_{i}(i=1,2,3)$, we obtain

$$
\langle\tilde{h}\rangle_{0}=\sum_{i=1}^{3} \int_{P_{i} C_{i} Q_{0} C_{i-1}} h d s .
$$

Given $\langle h\rangle_{i}, \bar{h}_{i},\langle\tilde{h}\rangle_{j}$, and $K_{j}(i, j=0,1,2, \ldots)$, we compute the area-weighted summation of total energy by

$$
E=\sum_{i} \frac{1}{2} g\langle h\rangle_{i}\left(\bar{h}_{i}+2 \bar{h}_{b, i}\right)+\sum_{j}\langle\tilde{h}\rangle_{j} K_{j}
$$

where the summations of $i$ and $j$ are taken for all hexagonal/pentagonal cells and all triangular cells, respectively.

Following Tomita et al. (2001), we evaluate

$$
R_{Z}(t)=\left|\frac{Z(t)-Z(0)}{Z(0)}\right|, \quad \text { and }
$$

$$
R_{E}(t)=\left|\frac{E(t)-E(0)}{E_{K}(0)}\right|
$$

as functions of time to check the conservations of potential enstrophy and total energy, respectively. Here, $E_{K}$ is the summation of kinetic energy, the second term on the righthand side of (48), and $t=0$ means the initial condition.

\section{Test results}

The SB-grid shallow-water model proposed in the previous section was subjected to some of the standard tests. We performed the test cases 2, 5, and 6 of Williamson et al. (1992) and the barotropically unstable, perturbed jet flow of Galewsky et al. (2004). Before those tests, we compared the response to a onepoint forcing of the SB-grid model with that of the Z-grid model to examine how the hyperviscosity works.

We adopted the three-stage Runge-Kutta time stepping scheme of Wicker and Skamarock (2002) except for the time-splitting. For example, we update (1) from time $t$ to $t+\Delta t$ by a three-stage calculation as follows:

$$
\begin{aligned}
h^{*} & =h^{t}+\frac{\Delta t}{3}\left[-\nabla \cdot\left(h^{t} \mathbf{v}^{t}\right)\right], \\
h^{* *} & =h^{t}+\frac{\Delta t}{2}\left[-\nabla \cdot\left(h^{*} \mathbf{v}^{*}\right)\right], \\
h^{t+\Delta t} & =h^{t}+\Delta t\left[-\nabla \cdot\left(h^{* *} \mathbf{v}^{* *}\right)\right] .
\end{aligned}
$$

Note that $h$ and $\mathbf{v}$ are integrated simultaneously in each substep.

We used the hexagonal/pentagonal meshes with 2562, 10242, $40962,163842,655362$, and 2621442 
(a) ZM

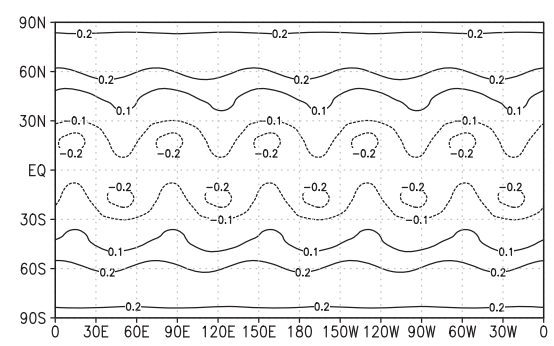

(d)

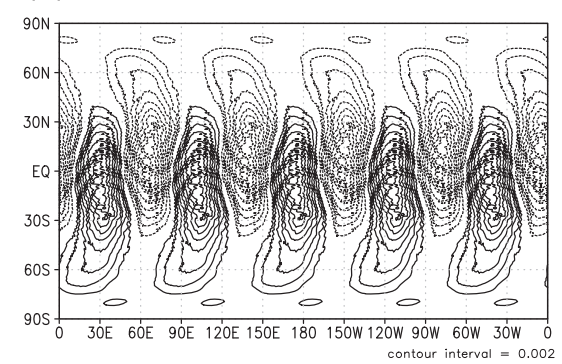

(b) SB $\nu_{8}=0$

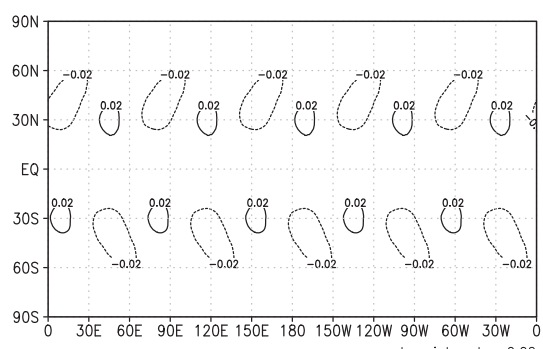

(e)

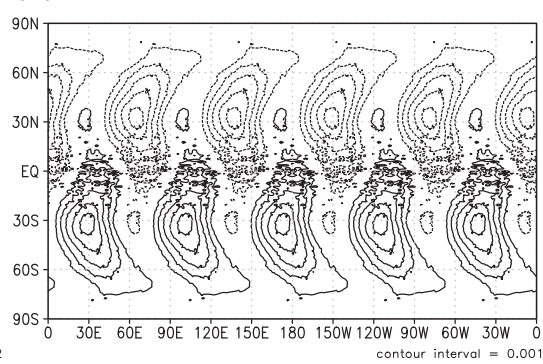

(c) SB

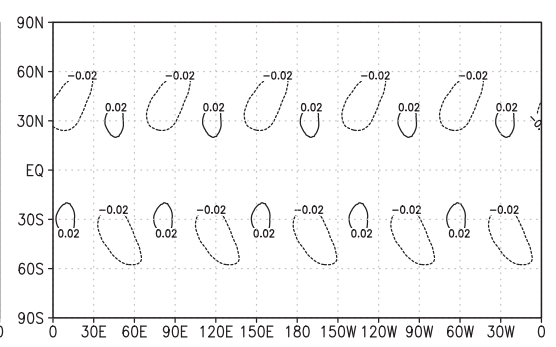

(f)

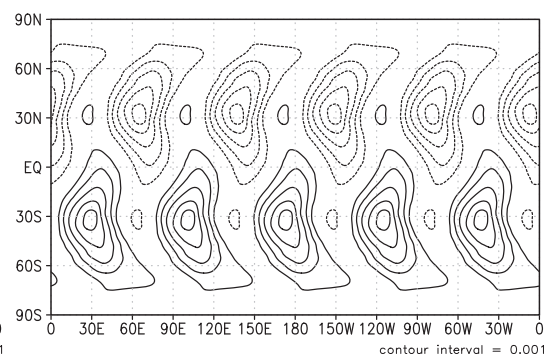

FIG. 13. The test case 2 of Williamson et al. (1992). Errors of (top) height and (bottom) meridional velocity after 365 days for H64. (a),(d) The ZM-grid model, and the SB-grid model (c),(f) with or (b),(e) without the hyperviscosity. The contour intervals are (a) $0.1 \mathrm{~m}$, (b), (c) $0.02 \mathrm{~m}$, (d) $0.002 \mathrm{~m} \mathrm{~s}^{-1}$, and (e),(f) $0.001 \mathrm{~m} \mathrm{~s}^{-1}$. The zero contour is omitted.

cells. Following Miura and Skamarock (2013), we call them H16, H32, H64, H128, H256, and H512 using the number of node points on each edge of the original icosahedron. They correspond to glevel-4, glevel-5, glevel-6, glevel-7, glevel-8, and glevel-9 in the notation of Tomita et al. (2001). For each resolution, the time interval was $\Delta t=480,240,120,60,30$, and $15 \mathrm{~s}$ respectively. We used the hyperviscosity of $n=4$ to reduce the smoothing effect in most of the tests, and $\nabla^{8}$ is computed by operating $\nabla^{2}$ four times. The hyperviscosity coefficient for each resolution was $\nu_{8}=1.6 \times$ $10^{37}, 1.3 \times 10^{35}, 1.0 \times 10^{33}, 7.0 \times 10^{30}, 4.0 \times 10^{28}$, and $1.0 \times 10^{26} \mathrm{~m}^{8} \mathrm{~s}^{-1}$, respectively. We will mention when we refer to simulations of $\nu_{8}=0.0 \mathrm{~m}^{8} \mathrm{~s}^{-1}$. The radius of Earth was $R_{e}=6.37122 \times 10^{6} \mathrm{~m}$, the angular speed of the daily rotation was $\Omega=7.292 \times 10^{-5} \mathrm{~s}^{-1}$, and the gravitational constant was $g=9.80616 \mathrm{~m} \mathrm{~s}^{-2}$ (Williamson et al. 1992). We rescaled the spatial operators defined for the unit sphere to Earth.

For a scalar $q$, accuracy was measured by the $L_{2}$ and $L_{\infty}$ norms, the definitions of which are

$$
\begin{aligned}
& L_{2}(q)=\frac{\sqrt{\sum_{i} A_{i}\left(\bar{q}_{i}-q_{i, \text { true }}\right)^{2}}}{\sqrt{\sum_{i} A_{i} q_{i, \text { true }}^{2}}} \text { and } \\
& L_{\infty}(q)=\frac{\max _{i}\left|\bar{q}_{i}-q_{i, \text { true }}\right|}{\max _{i}\left|q_{i, \text { true }}\right|},
\end{aligned}
$$

where $\bar{q}_{i}$ is the mean value computed for the $i$ th hexagonal/pentagonal cell, and $q_{i, \text { true }}$ is the exact solution at the node point of the $i$ th hexagonal/pentagonal cell. The summation is computed or the maximum is evaluated for all of the hexagonal/pentagonal cells. When no analytic solution was available, we used the solutions computed by the NCAR Spectral Transform Shallow Water Model (STSWM; Jakob-Chien et al. 1995) with T639 horizontal resolution as substitutions of the true solutions. We note that STSWM results were sensitive to setttings of the time interval and the hyperviscosity coefficient. We used $\Delta t=30 \mathrm{~s}$ for the case 5 and $\Delta t=15 \mathrm{~s}$ for the case 6 , respectively. The coefficient of the fourth-order hyperviscosity was $\nu_{4}=2.0 \times 10^{11} \mathrm{~m}^{4} \mathrm{~s}^{-1}$ commonly.

\section{a. Response to a one-point forcing}

At the beginning, we examine the response of the SBgrid model to a one-point forcing given at the midpoint between a pair of midlatitude pentagons in the Northern Hemisphere. The initial conditions were $h=(2.94 \times$ $\left.10^{4}\right) / g \mathrm{~m}$ and $\mathbf{v}=0 \mathrm{~m} \mathrm{~s}^{-1}$ everywhere, except for one hexagonal cell at about $\left(31.7^{\circ} \mathrm{N}, 36^{\circ} \mathrm{E}\right)$, where $h=1.1 \times$ $\left(2.94 \times 10^{4}\right) / g \mathrm{~m}$. The bottom boundary was $h_{b}=0 \mathrm{~m}$ uniformly. We performed three different simulations for the H64 resolution. The first is the control run with the second-order accurate gradient, (33), and with the $\nabla^{8}$ hyperviscosity (Fig. 11c). The second run uses the first-order accurate gradient similarly to Miura (2017) 
(a) $\nu_{8}=0$

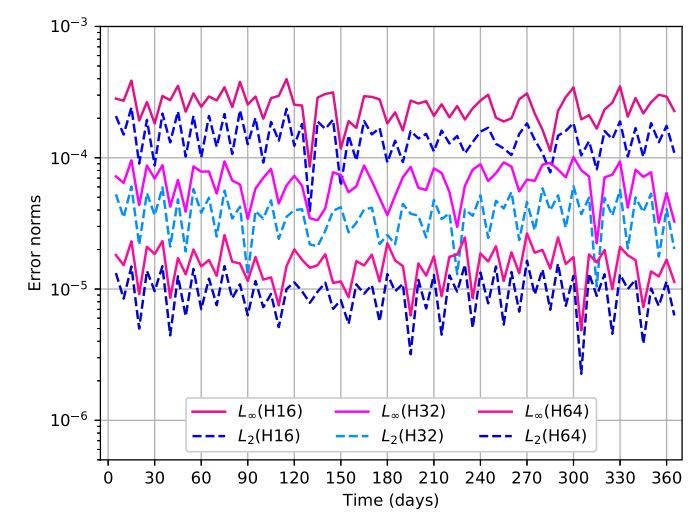

(b)

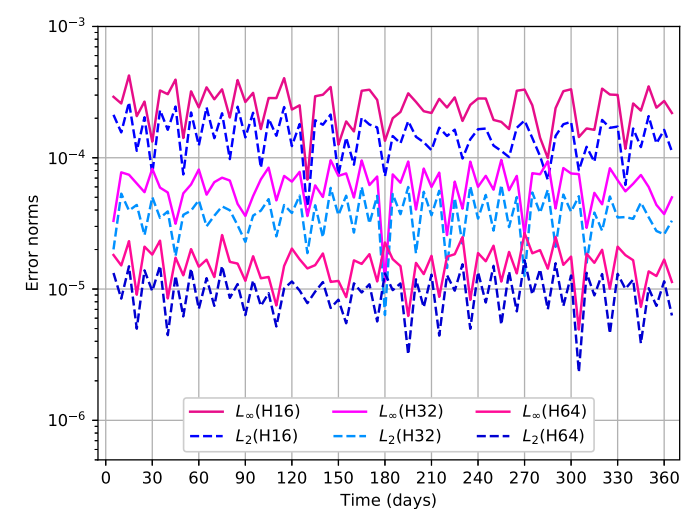

FIG. 14. The test case 2. As in Fig. 4, but for the SB-grid model (b) with or (a) without the hyperviscosity. The H64 results appear almost identical, but raw numbers are different.

(Fig. 11b). The last is the run without the hyperviscosity (Fig. 11d). We regard a simulation by a Z-grid model developed following Heikes and Randall (1995) as a reference (Fig. 11a).

One day after the initial time, geostrophic adjustment and gravity waves propagating away from the initial perturbation are adequately reproduced when velocity fields are synchronized (Figs. 11b,c). Figure 11b is more consistent with Fig. 11a than Fig. 11c. This is because the second-order accurate gradient, which is introduced for better geostrophic balance, modifies the dispersion relation although this side effect appears not significant. Without the synchronization of velocity, gravity waves do not radiate away efficiently (Fig. 11d), and smallerscale noises are evident in the velocity field (Fig. 12a). The hyperviscosity successfully removes them (Fig. 12b). In this test, the geostrophic balance is not significant in magnitude comparing to gravity wave motions since the initial velocity is uniformly zero, and thus, the constraint (7) is not effective in synchronizing the velocity fields, and the hyperviscosity is needed.

\section{b. Global steady-state nonlinear zonal gestrophic flow (test case 2)}

In the test case 2 of Williamson et al. (1992), the initial conditions of the fluid depth, and the eastward and northward components of velocity at longitude $\lambda$ and latitude $\theta$ were

$$
g h=g h_{0}-\frac{u_{0}}{2}\left(2 R_{e} \Omega+u_{0}\right)(\sin \theta \cos \alpha-\cos \lambda \cos \theta \sin \alpha)^{2},
$$

$$
\begin{aligned}
& u=u_{0}(\cos \theta \cos \alpha+\cos \lambda \sin \theta \sin \alpha), \quad \text { and } \\
& v=-u_{0} \sin \lambda \sin \alpha
\end{aligned}
$$

where $g h_{0}=2.94 \times 10^{4} \mathrm{~m}^{2} \mathrm{~s}^{-2}$, and $u_{0}=2 \pi R_{e} /(8.64 \times$ $\left.10^{4} \times 12\right) \mathrm{m} \mathrm{s}^{-1}$. The Coriolis parameter was

$$
f=2 \Omega(\sin \theta \cos \alpha-\cos \lambda \cos \theta \sin \alpha) .
$$

The parameter $\alpha$ is the angle between the axis of the flow rotation and the polar axis of the sphere. We analyze results of $\alpha=0$. The bottom surface height was $h_{b}=0 \mathrm{~m}$ everywhere. Simulations of the period of 365 days were performed for the H16, H32, and H64 resolutions to examine whether the SB-grid model is as stable as an Agrid model (Tomita et al. 2001) and a C-grid model (Ringler et al. 2010). We examine the convergence of the error norms on day 5 .

Comparing to a ZM-grid model on H64 with $\nu_{8}=1.0 \times$ $10^{33} \mathrm{~m}^{4} \mathrm{~s}^{-1}$ hyperviscosity, the magnitudes of errors of height and meridional velocity are about one-tenth smaller

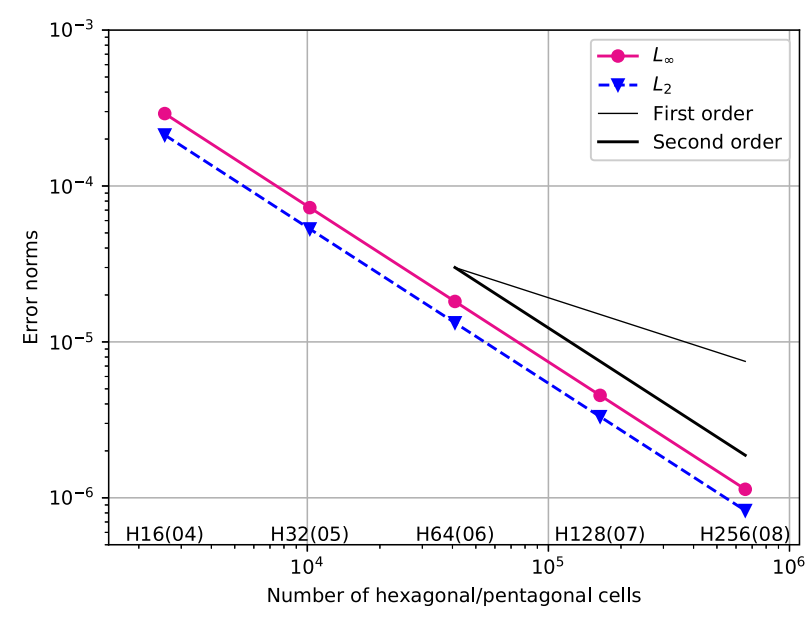

FIG. 15. The test case 2. Dependences of $L_{2}(h)$ and $L_{\infty}(h)$ after 5 days on the mesh refinement. The thick and thin solid lines are references of the second-order and first-order convergences, respectively. 
(a)
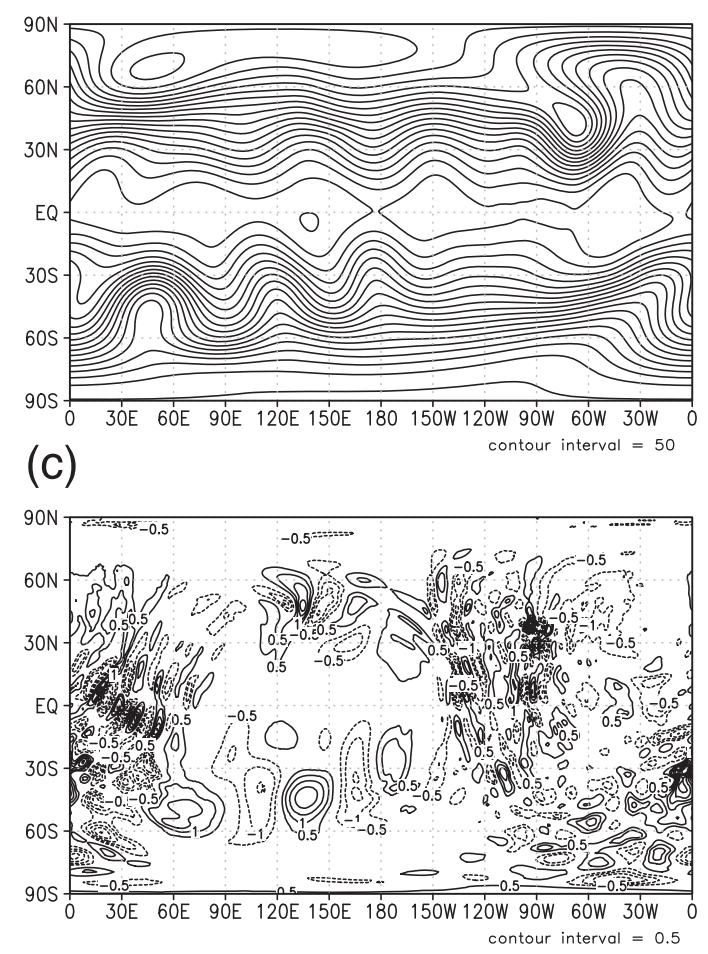

(b)

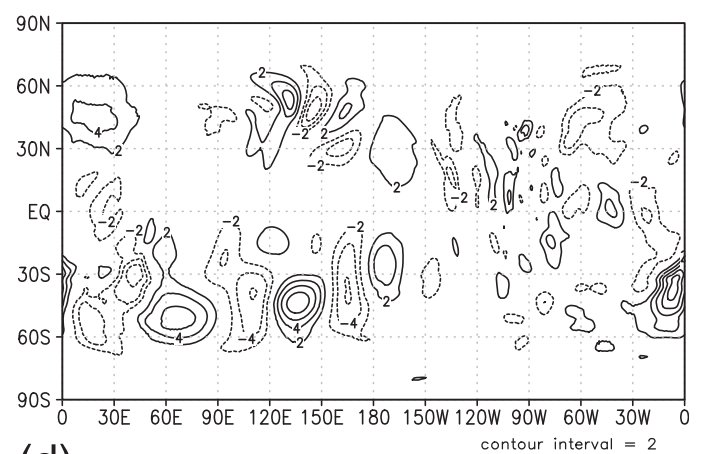

(d)

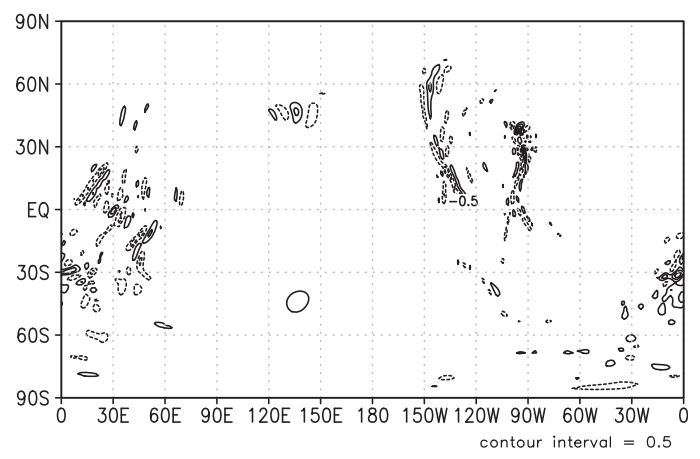

FIG. 16. The test case 5 of Williamson et al. (1992). (a) Height after 15 days for H64, and height differences from the T639 solution after 15 days for (b) H64, (c) H128, and (d) H256 (glevels-6, 7, and 8). The contour intervals are (a) 50, (b) 2, and (c),(d) $0.5 \mathrm{~m}$. The zero contour is omitted in (b)-(d).

for the SB-grid model even when $\nu_{8}=0.0 \mathrm{~m}^{4} \mathrm{~s}^{-1}$ (Fig. 13). This difference is also detectable in the time evolution of $L_{2}(h)$ and $L_{\infty}(h)$ by comparing Figs. 4 and 14. For all of the H16, H32, and H64 resolutions, the error norms of the SB-grid simulations did not amplify during the integrations though with small fluctuations, while those of the ZM-grid ones increased gradually in later periods. This result confirms that the SB-grid can make stable long-term simulations as those demonstrated by an A-grid model (Tomita et al. 2001) and a C-grid model (Ringler et al. 2010). Without the synchronization of velocity, meridional wind field is noisy especially near the equator (Fig. 13e), where the geostrophic balance is not significant enough to dominate the nonlinear terms. The hyperviscosity effectively removed those smaller-scale noises without changing large-scale features (Figs. 13e,f) and the magnitudes of the error norms (Fig. 14).

The convergence rate of $L_{2}(h)$ and $L_{\infty}(h)$ on day 5 is almost second order (Fig. 15). The magnitudes of the error norms are about a quarter of those of the A-grid model of Tomita et al. (2001) for each resolution. This convergence property is a distinct advantage of the SBgrid model to the $\mathrm{C}$-grid model and the $\mathrm{Z}$-grid model. As it was pointed out by Peixoto (2016), $L_{\infty}(h)$ does not converge, and $L_{2}(h)$ converges at nearly the first order as resolution increases in the C-grid model of Ringler et al. (2010). Peixoto (2016) proposed a method to improve the convergence rate at the expense of the stationary geostrophic mode. $L_{\infty}(h)$ of the SB-grid model appears even smaller than the C-grid model of Peixoto (2016).

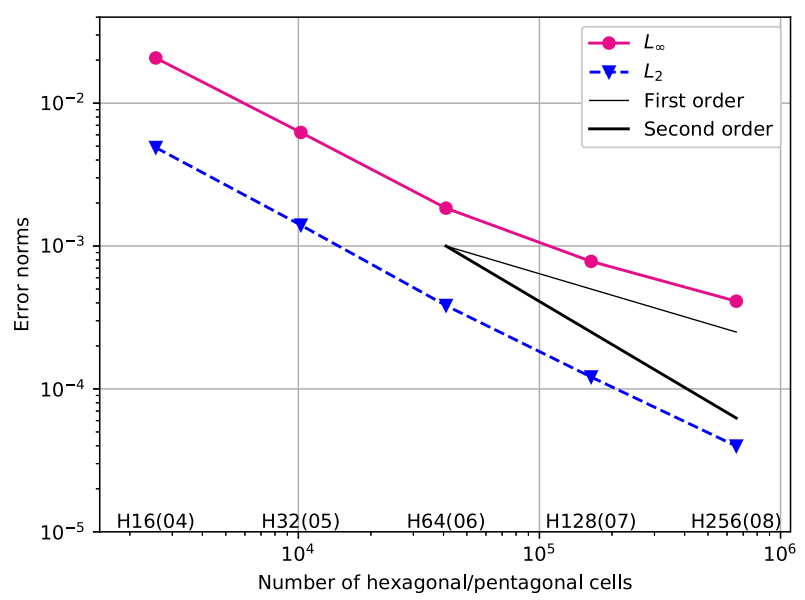

FIG. 17. As in Fig. 15, but for after 15 days in the test case 5 . 
(a) $\nu_{8}=0$
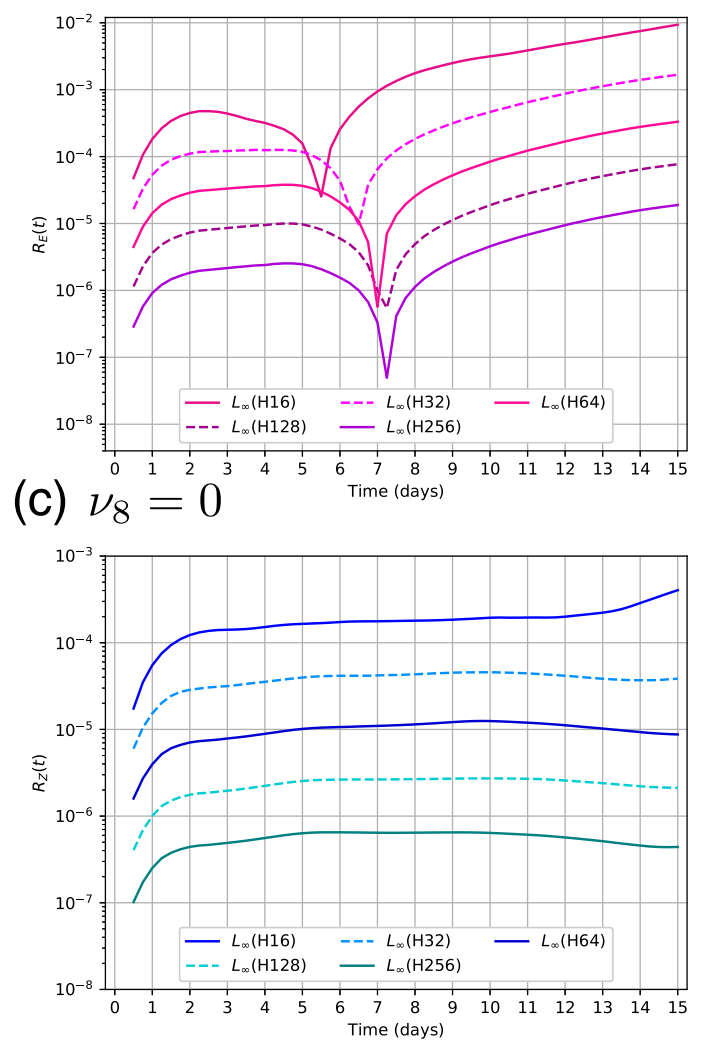

(b)

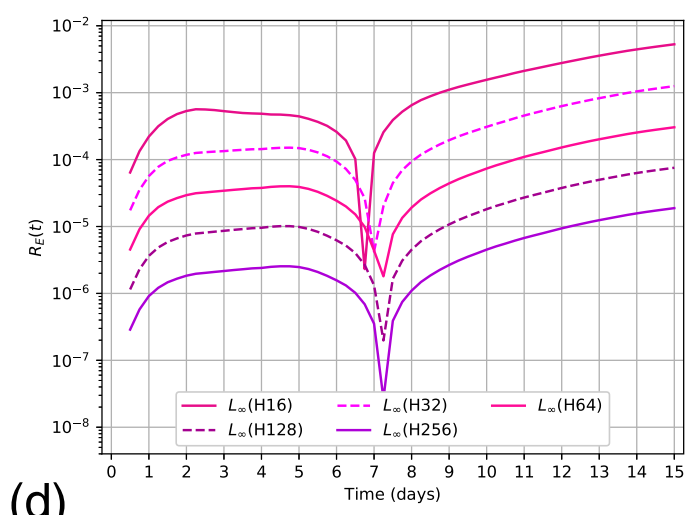

(d)

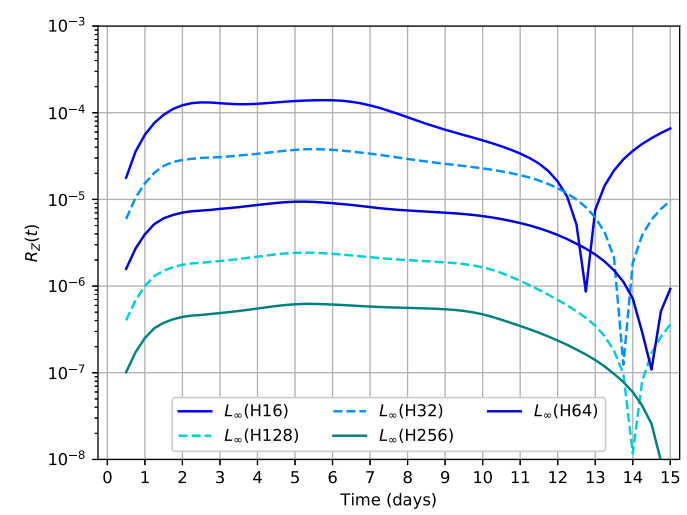

FIG. 18. The test case 5. Temporal variations of (a),(b) $R_{E}(t)$ and (c),(d) $R_{Z}(t)$; (b),(d) with or (a),(c) without the hyperviscosity.

The convergence rate of $L_{\infty}(h)$ of the Z-grid model is roughly the first order even when a grid optimization is used (Miura 2004; Eldred 2015).

\section{c. Zonal flow over an isolated mountain (test case 5)}

The initial conditions of the test case 5 of Williamson et al. (1992) were given by (54)-(56) with $h_{0}=5960 \mathrm{~m}$ and $u_{0}=20 \mathrm{~m} \mathrm{~s}^{-1}$. We used $\alpha=0$. A mountain centered at $\left(\lambda_{c}, \theta_{c}\right)=(3 \pi / 2, \pi / 6)$ was set by

$$
h_{b}=h_{b_{0}}\left(1-\frac{r}{r_{0}}\right)
$$

where $h_{b_{0}}=2000 \mathrm{~m}, r_{0}=\pi / 9$, and $r^{2}=\min \left[r_{0}^{2},\left(\lambda-\lambda_{c}\right)^{2}+\right.$ $\left.\left(\theta-\theta_{c}\right)^{2}\right]$.

The fluid surface height on day 15 of the H64 simulation (Fig. 16a) appears less diffusive and more accurate than Fig. 13 of Tomita et al. (2001), which used the same number of hexagonal/pentagonal cells (glevel-6). Differences from STSWM solutions are about $10 \mathrm{~m}$ for the SB-grid model and about $25 \mathrm{~m}$ for the A-grid model of Tomita et al. (2001). Note that the settings of the spectral models are different: we used T639 horizontal resolution as a reference, but Tomita et al. (2001) adopted T213. The convergence rate of $L_{2}\left(h_{a}\right)$ and $L_{\infty}\left(h_{a}\right)$ becomes lower as resolution increases and looks asymptotically approaching the first order (Fig. 17). Because of a discontinuous transition at the foot of the mountain, $h_{b}$ is not differentiable along the perimeter of the mountain, and thus, accuracy inevitably degrades to first order at most not only in icosahedral grid models but also in spectral models. As consistent with this consideration, we see that decreases in differences between the SB-grid model and the T639 result are slower around the mountain (Figs. 16b-d). The magnitudes of $L_{2}\left(h_{a}\right)$ and $L_{\infty}\left(h_{a}\right)$ on day 15 are smaller than an A-grid model (Tomita et al. 2001) and comparable to a C-grid model (Ringler et al. 2010).

Total energy and potential enstrophy are not conserved formally in the SB-grid model, but conservation properties are competitive with the other models. Time evolutions of $R_{E}$ and $R_{Z}$ of the SB-grid model are similar to the A-grid model of Tomita et al. (2001) although with smaller magnitudes (Figs. 18b,d). Both total energy 


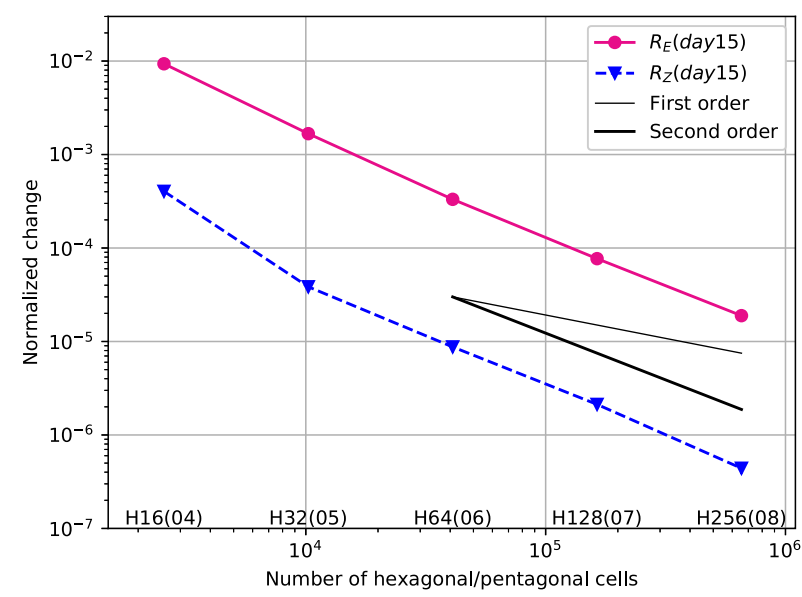

FIG. 19. The test case 5. Dependences of $R_{E}(t)$ and $R_{Z}(t)$ after 15 days on the mesh refinement.

and potential enstrophy increase gradually until about day 6 and turn to decrease monotonically after that. Without the hyperviscosity, the potential enstrophy is well conserved for the simulation period (Fig. 18c) though the kinetic energy conservation is not strongly sensitive to the hyperviscosity (Fig. 18a). The SB-grid model is inferior to the C-grid model of Ringler et al.
(2010) about total energy conservation by definition since the $\mathrm{C}$-grid model conserves total energy formally. About potential enstrophy conservation, the SB-grid model is better than the energy conserving C-grid model, comparing Figs. $18 \mathrm{c}$ or $18 \mathrm{~d}$ with Fig. 9 of Ringler et al. (2010). The convergence rate of $R_{E}$ and $R_{Z}$ at day 15 is roughly the second order when the hyperviscosity is not used (Fig. 19).

\section{d. Rossby-Haurwitz wave (test case 6)}

The initial conditions of the Rossby-Haurwitz wave were set following the test case 6 of Williamson et al. (1992), and we do not include details here. Note that there is a limitation in the discussion about the error convergences since this case is dynamically unstable (Thuburn and Li 2000).

The fluid surface on day 14 of H64 (Fig. 20a) looks less diffusive and more accurate than Fig. 20b of Tomita et al. (2001). The H64 result of the SB-grid appears comparable to their glevel 7 (H128) result. By comparing Fig. 21 and Figs. 21a and 21b of Tomita et al. (2001), it is confirmed that $L_{2}(h)$ and $L_{\infty}(h)$ are smaller in the SB-grid model than their A-grid model, but note again that the settings of the spectral models are different. The convergence rate of those norms on day 14 (a)

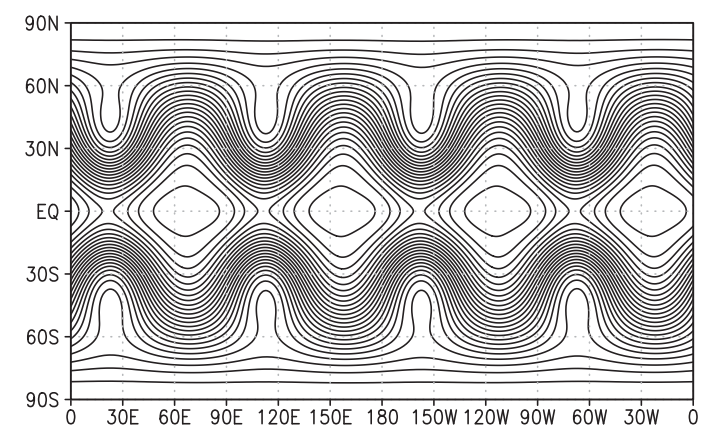

(c)

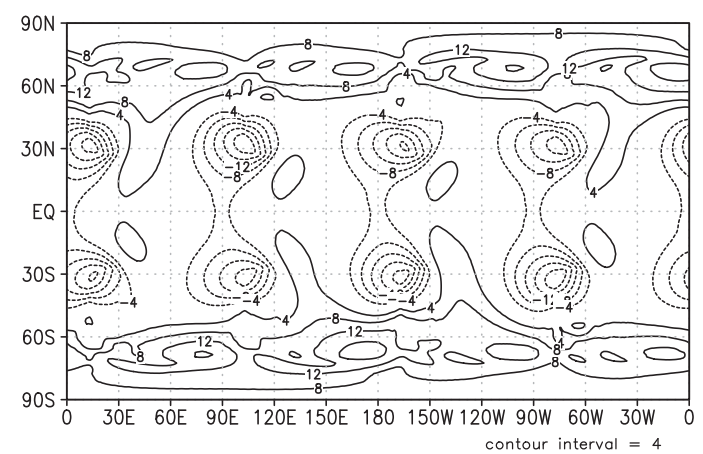

(b)

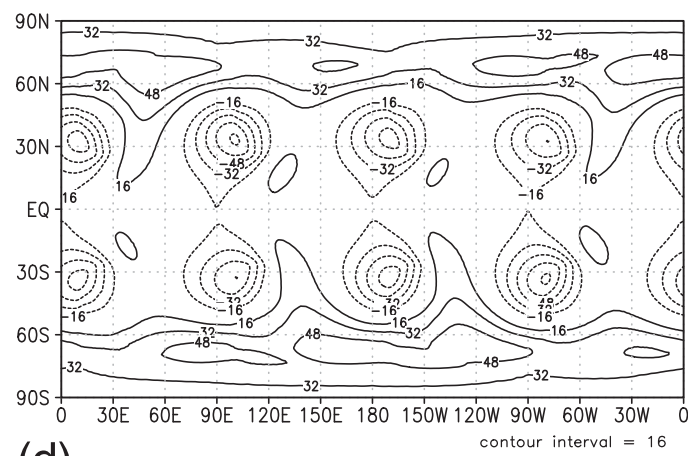

(d)

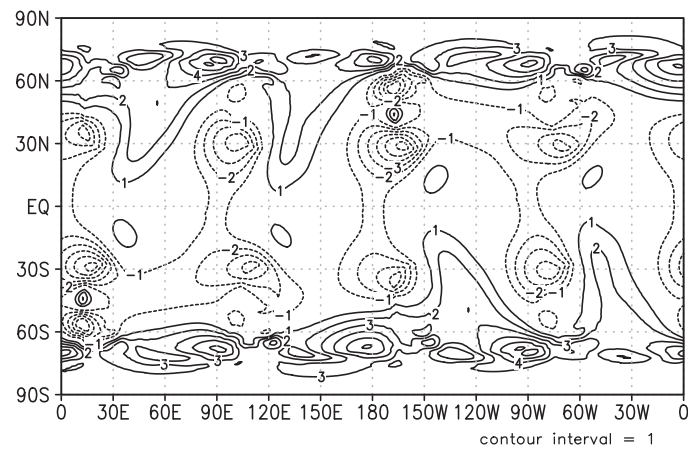

FIG. 20. As in Fig. 16, but for after 14 days in the test case 6 of Williamson et al. (1992). The contour intervals are (a) 100 , (b) 16, (c) 4, and (d) $1 \mathrm{~m}$. The zero contour is omitted in (b)-(d). 


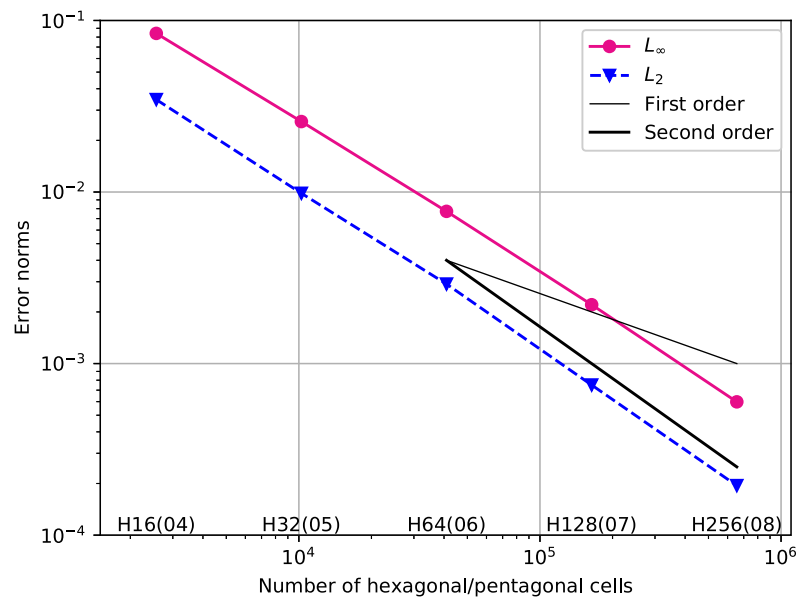

FIG. 21. As in Fig. 15, but for after 14 days in the test case 6.

is nearly the second order. The maximum magnitudes of differences between the SB-grid model and STSWM are about 80, 20, and $6 \mathrm{~m}$ for H64, H128, and H256, respectively. This result suggests that convergence to the STSWM solution slows down between H128 and $\mathrm{H} 256$. However, the STSWM solution is not the exact solution but a reference one, and thus, it is unclear whether this degradation of convergence is due to the SB-grid model or due to the STSWM model.

Comparing Fig. 21 with Fig. 10 of Ringler et al. (2010) and Fig. 13 of Peixoto (2016), we find that the SB-grid model is less accurate than the C-grid model for H16, $\mathrm{H} 32$, and H64. This lower accuracy is probably due to the lower resolution of the nonlinear gradient and curl operators. Their horizontal resolutions are almost the same as those of the A-grid model since they use the stencils of the larger hexagonal shape (Fig. 10). About the C-grid models, convergence rate of $L_{2}(h)$ and $L_{\infty}(h)$ becomes almost first order as resolution increases.
Because of this slow convergence of the C-grid models, the error norms of the SB-grid model are only slightly larger for $\mathrm{H} 128$ and are even smaller for $\mathrm{H} 256$.

Figure 22 shows the time evolutions of $R_{E}$ and $R_{Z}$. Both total energy and potential enstrophy increase in earlier periods and become to decrease in later periods. We cannot compare the SB-grid model with the A-grid model nor the C-grid model since neither Tomita et al. (2001), Ringler et al. (2010), nor Peixoto (2016) showed a corresponding figure. Comparing to the results produced by higher-order finite-volume models using the multimoment reconstruction (Chen et al. 2014) or the higher-order reconstruction with larger spatial stencils on the cubed-sphere mesh (Ullrich et al. 2010), temporal variations of total energy and potential enstrophy are reasonable.

\section{e. Barotropically unstable jet (Galewsky et al. 2004)}

Galewsky et al. (2004) proposed a test of a barotropically unstable jet with an initial perturbation. It is a tough test for a lower-order model on a non-latitudelongitude grid (Weller et al. 2012) since a balance of height and velocity fields is unstable and can be readily destroyed by numerical errors in initial conditions and truncation errors.

There are larger bents of the axis of the jet near $90^{\circ}$ and $150^{\circ} \mathrm{E}$ after 6 days for H128 (Fig. 23a), but they are not observed for $\mathrm{H} 256$ and H512 (Figs. 23b,c). This result indicates that the $\mathrm{H} 128$ resolution suffers from the influence of the wavenumber five structure of the icosahedral mesh. Figure 11a of Weller et al. (2012) showed that their C-grid model could reproduce a better result with the same number of hexagonal/pentagonal cells. The lower-resolution nonlinear terms are possibly responsible for the distortion of the solution of the SB-grid model at this resolution, but Fig. 15 of Peixoto (2016) (a)

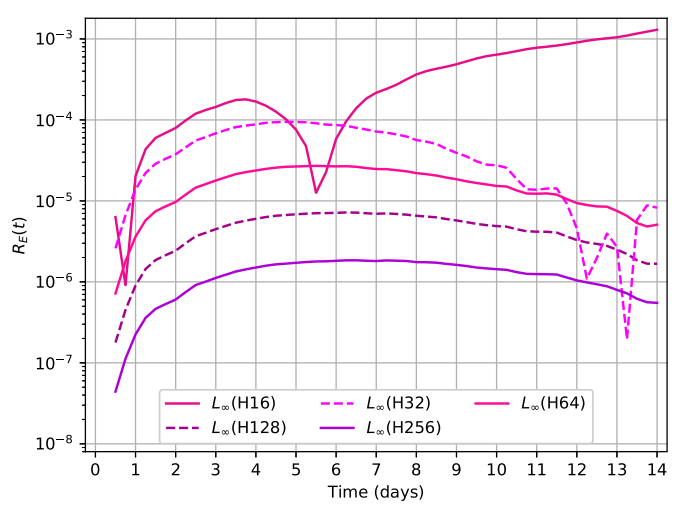

(b)

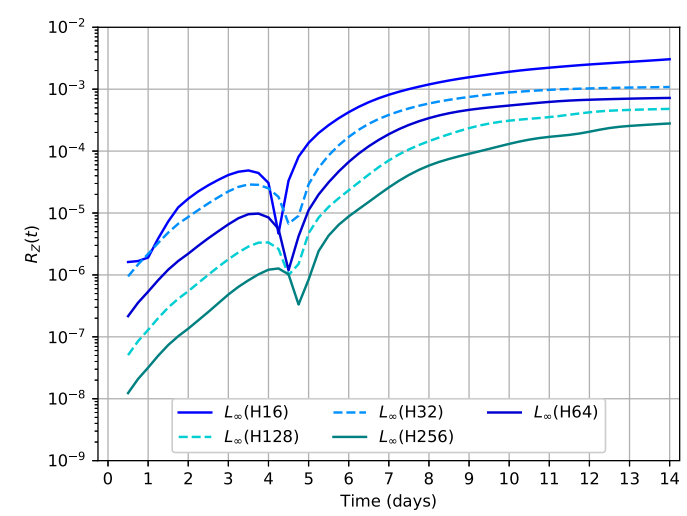

FIG. 22. As in Fig. 18, but for the test case 6 with the hyperviscosity. 

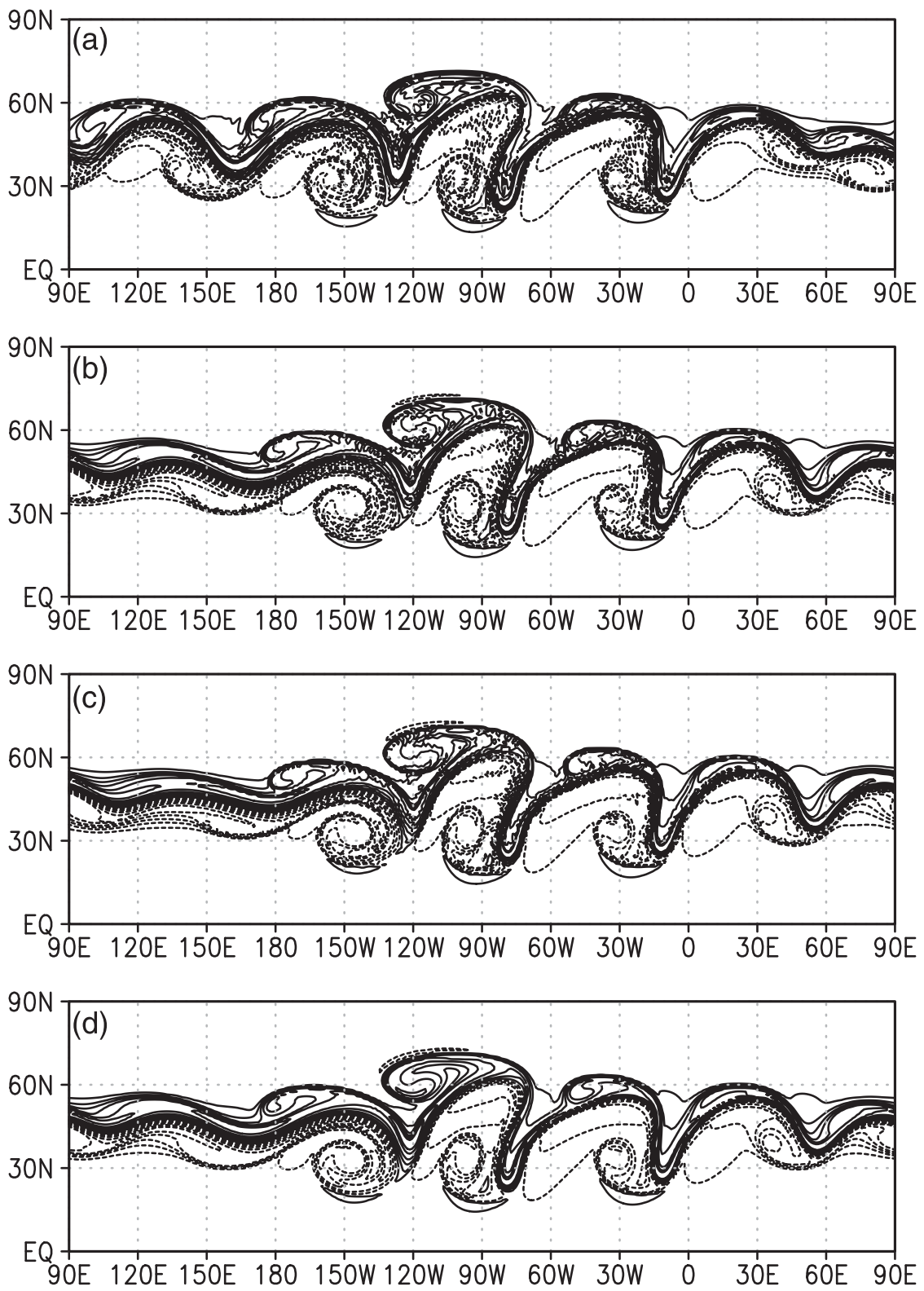

FIG. 23. The barotropically unstable jet test of Galewsky et al. (2004). Relative vorticity after 6 days for (a) H128, (b) H256, and (c) H512 (glevels-7, 8, and 9) with the $\nabla^{8} \mathbf{v}$ hyperviscosity and for (d) H256 with the $\nabla^{4} \mathbf{v}$ hyperviscosity. Similar to Ullrich et al. (2010), contour lines are in increments of $2.0 \times 10^{-5} \mathrm{~s}^{-1}$ from $-1.1 \times 10^{-4}$ to $-0.1 \times 10^{-4} \mathrm{~s}^{-1}$ (dashed) and from $0.1 \times 10^{-4}$ to $1.1 \times 10^{-4} \mathrm{~s}^{-1}$ (solid). The zero line is omitted.

showed that a C-grid model was still sensitive to a choice of spatial discretization and grid optimizations for this resolution. Results of $\mathrm{H} 256$ and $\mathrm{H} 512$ are quite similar to solutions obtained by the higher-order models (Chen et al. 2014; Ullrich et al. 2010).

Small-scale noises are apparent for the results with the $\nabla^{8}$ hyperviscosity (Figs. 23a-c). Those noises can be smoothed out if we use the $\nabla^{4}$ hyperviscosity with $\nu_{4}=$ $6 \times 10^{13} \mathrm{~m}^{4} \mathrm{~s}^{-1}$ for H256 (Fig. 23d). The reason for those noises is filamentations of relative vorticity but not a decoupling of velocity fields as in Figs. 11d and 13e. Figure 24 compares relative vorticity fields after 6 days for $\mathrm{H} 512$ with the $\nabla^{8}$ or $\nabla^{4}$ hyperviscosity term. The hyperviscosity coefficient for the fourth-order Laplacian was $\nu_{4}=6 \times 10^{12} \mathrm{~m}^{4} \mathrm{~s}^{-1}$. Sharper structures are computed with $\nabla^{8}$ than with $\nabla^{4}$. The $\nabla^{8}$ result shows smaller-scale ripples, which do not exist in the $\nabla^{4}$ result. We can observe filament structures similar to Fig. 24a 

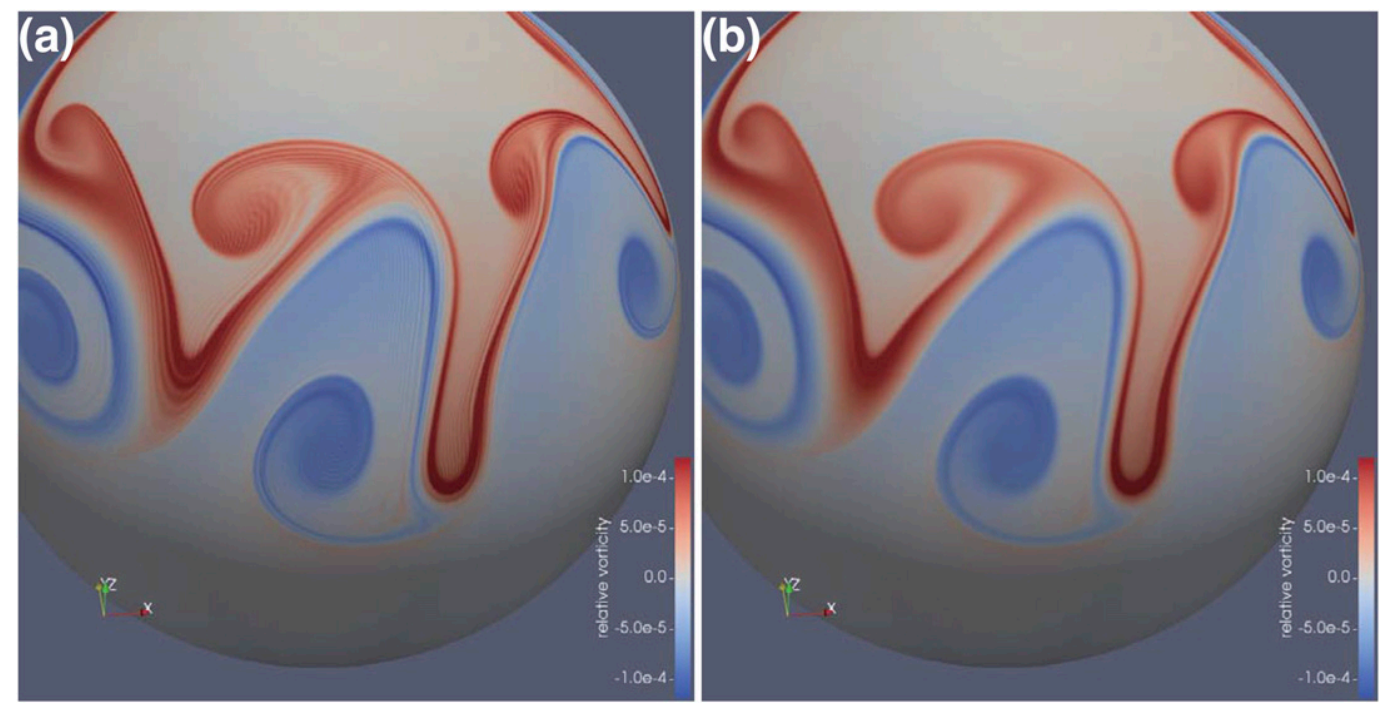

FIG. 24. The barotropically unstable jet test. Relative vorticity after 6 days for H512 (glevel-9) with (a) the $\nabla^{8} \mathbf{v}$ hyperviscosity and (b) the $\nabla^{4} \mathbf{v}$ hyperviscosity.

in the C-grid model results of Weller et al. (2012) and Peixoto (2016).

\section{f. Test results without a grid optimization}

In the tests shown so far, the model integrations were performed on an optimized hexagonal/pentagonal grid. Here, we examine results of the test cases 2, 5, and 6 of Williamson et al. (1992) computed on the nonoptimized grid. We used the barycentric-type mesh again to ensure the second-order accuracy of the cell averaging in (33).

In the test case 2, the SB-grid model stably ran for 365 days (Fig. 25a). Magnitudes of the error norms (Fig. 25b) are roughly the same as those on the optimized meshes (Fig. 15). This is also the case for the test cases 5 (Fig. 25c) and 6 (Fig. 25d). This feature that accuracy and stability are rarely sensitive to a choice of with or without grid optimizations is a distinct advantage of the SB-grid model against the A-grid model (Tomita et al. 2001) and the C-grid model (Ringler et al. 2010; Peixoto 2016).

\section{Summary and conclusions}

We have applied the hexagonal synchronized B grid (Miura 2017), which provides a pathway to avoid a computational model problem of the ZM grid (Ringler and Randall 2002a,b), to solve the shallow-water equations on the spherical icosahedral mesh with the following revisions. A second-order accurate gradient operator was developed to improve representations of the geostrophic balance. A hyperviscosity term was added to the momentum equations to synchronize evolutions of the velocity fields. We also proposed a quadratic reconstruction method that satisfies an integral constraint precisely for the target cell and approximately for the surrounding cells in appendix A. A test of the response to a one-point forcing has demonstrated that the hyperviscosity successfully realizes matched evolutions of the velocity fields. We have performed the test cases 2, 5, and 6 of Williamson et al. (1992) and the barotropically unstable jet test of Galewsky et al. (2004) to examine the SB-grid shallowwater model. The test results were compared with the results of an A-grid model of Tomita et al. (2001) and C-grid models of Ringler et al. (2010), Weller et al. (2012), and Peixoto (2016).

In the test case 2, the SB-grid model ran for 365 days stably even without the hyperviscosity (Fig. 14). The convergence rate of the error norms was approximately the second order (Fig. 15). The stability was comparable to the A-grid model and the $\mathrm{C}$-grid model, and the accuracy was better than those models. In the test case 5 , the magnitudes of the difference from a spectral model solution of the SB-grid model were smaller than the A-grid model and were comparable to the C-grid model (Figs. 16 and 17). Concerning the total energy conservation, the SB-grid model was inferior to the C-grid model, which conserves total energy by definition, but was superior to the A-grid model (Figs. 18a,b). The conservation of potential enstrophy of the SB-grid model was better than the A-grid and Cgrid models (Figs. 18c,d). In the test case 6, the magnitudes of the error norms of the SB-grid model were larger than the C-grid model for H16, H32, and H64 (Fig. 21). However, the convergence of the error norms 
(a)
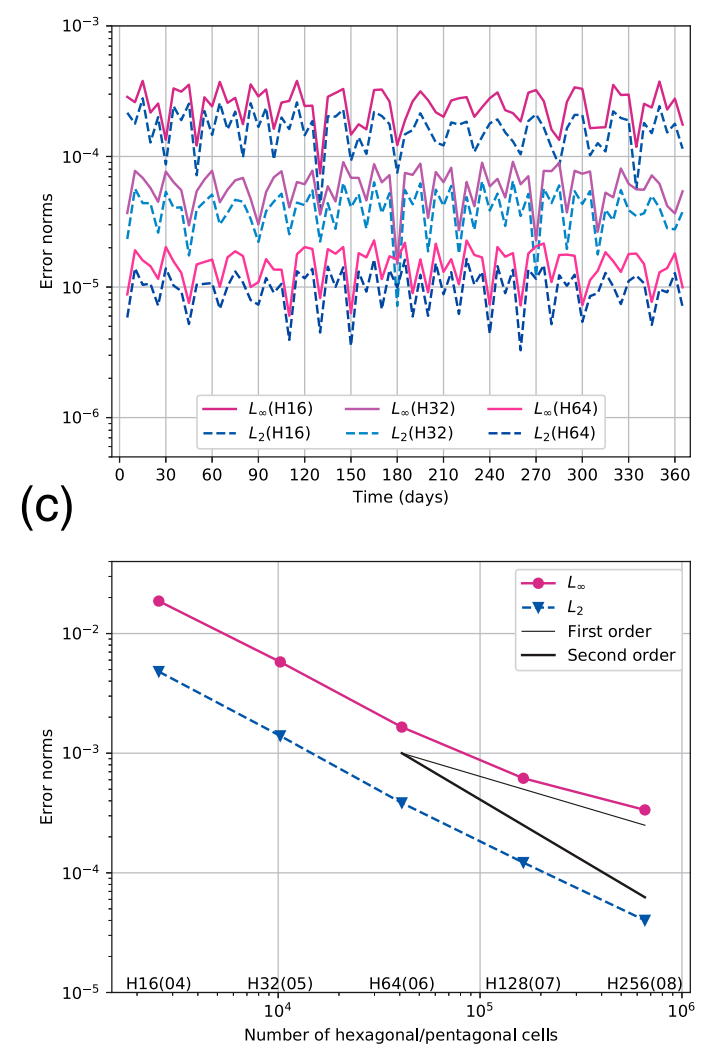

(b)
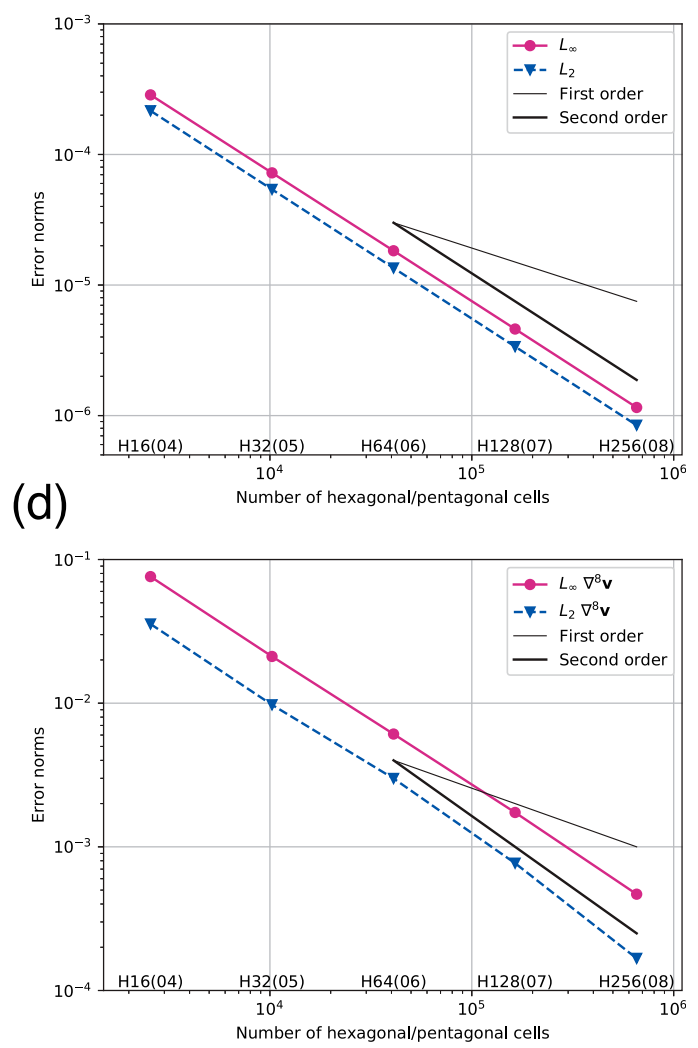

FIG. 25. As in (a) Fig. 14b, (b) Fig. 15, (c) Fig. 17, and (d) Fig. 21, but for the nonoptimized grid.

was faster for the SB-grid model than for the C-grid model, and the error magnitudes were almost comparable for H128, and those of the SB-grid model became smaller for H256. In the barotropically unstable jet test, the SB-grid model suffered from the wavenumber five structure of the icosahedral grid for H128 but reproduced similar solutions obtained by the higher-order models (Chen et al. 2014; Ullrich et al. 2010) for H256 and H512 (Fig. 23).

Based on these investigations, it is concluded that the $\mathrm{SB}$ grid is an attractive alternative to the conventional A grid (Tomita et al. 2001) and is competitive with the C grid (e.g., Ringler et al. 2010) on the spherical icosahedral grid. One good point of the SB grid is that both accuracy and stability are weakly sensitive to whether a grid optimization is applied or not. A grid optimization was a time-consuming task when very high-resolution simulations were performed by NICAM (Miura et al. 2007; Miyamoto et al. 2013), and thus, this property will help us when we proceed to further high-resolution simulations in the future.

Computational costs of the SB grid to update velocity vectors are slightly less than twice as much as the A grid when the $\nabla^{4}$ hyperviscosity is used since the number of velocity points is double. Although the configuration of the second-order accurate gradient (33) appears complicated, we can straightforwardly transform it into a simple form. For example, the $\mathbf{e}_{1}$ component of $\nabla h_{a}$ can be computed by

$$
\mathbf{e}_{1} \cdot \nabla h_{a}=\sum_{j=1}^{12 \operatorname{or} 11} b_{e_{1}, j} h_{j}
$$

where $j$ refers to the hexagonal nodes that surrounds the target point. Note that $b_{e_{1}, j}$ is time-independent, and thus, we can precompute and store them before the time loop. Computations associated with the quradratic reconstruction are represented by $b_{e_{1}, j}$ and do not appear explicitly inside the time loop. For the shallow-water system, the twice as much computational cost is nonnegligible. For the atmospheric model, however, it is expected that this difference is minor since all of the prognostic and diagnostic variables except for the horizontal components of velocity are defined at the cell nodes.

One of the concerns of the SB grid approach is in the curl and gradient operators for the nonlinear terms, which are configured using a larger hexagonal cell 
(Fig. 10). These operators are needed to avoid nonlinear interactions of side-by-side velocity points but are probably the reason for the larger error than the C-grid model in the test case 6 at lower resolutions. One of the other possible approaches to managing the mismatch of the degrees of freedom between mass points and velocity points is the mixed finite element method, which has successfully worked in the C-grid model on the spherical icosahedral mesh (Cotter and Shipton 2012; Thuburn and Cotter 2015). Recently, Shipton et al. (2018) achieved a significant improvement in accuracy by using higher-order basis functions. If the mixed finite element method is successfully extended to the hexagonal/pentagonal B grid, not only similar improvements may be expected, but also consistency between physical variables may be enhanced.

Another concern is that the tests of the SB-grid model is limited to the shallow-water system in this work. One of the motivations to develop the SB grid was that a 3D atmospheric model using the $\mathrm{ZM}$ grid needed a too strong hyperviscosity to conduct realistic simulations. This 3D ZM-grid model suffered from severe checkerboard noises in the velocity fields near the mountains. It is expected that those noises around mountains are reduced substantially for the SB grid since the spurious inertial mode in the velocity fields of the ZM grid does not exist. The next step is to develop a 3D atmospheric model using the SB grid to examine whether this conjecture is true.

Acknowledgments. Hiroaki Miura thanks for his former supervisor, Prof. Masahide Kimoto, for patiently awaited the finish of this work. He is also grateful to Almut Gassmann, Pedro S. Peixoto, Sergey Danilov and two anonymous reviewers for constructive suggestions and encouragements. This work was supported by JSPS Grant-in-Aid for Scientific Research (B) 25287119 and JSPS Grant-in-Aid for Scientific Research (B) 16H04048.

\section{APPENDIX A}

\section{A Quadratic Reconstruction Constrained by Cell Integrals}

We summarize the method of a quadratic reconstruction that is used in the flux-divergence and gradient operators. Given cell integrals of a scalar $q$,

$$
\langle q\rangle_{i}=\int_{A_{i}} q(\mathbf{x}) d A
$$

for all hexagonal/pentagonal cells $(i=0,1,2, \ldots)$, we want to approximate the distribution $q(\mathbf{x})$ inside the 0th cell by a quadratic polynomial $\tilde{q}_{0}(\mathbf{x})$ that satisfies

$$
\langle q\rangle_{0}=\int_{A_{0}} \tilde{q}_{0}(\mathbf{x}) d A
$$

for the target cell, and approximates

$$
\langle q\rangle_{i}=\int_{A_{i}} \tilde{q}_{0}(\mathbf{x}) d A
$$

for the surrounding cells. The flow of the derivation is similar to Miura and Skamarock (2013) and Miura (2013), but here we need to consider the area integral over the surrounding cells.

We consider a tangential plane that is spanned about the node point $P_{0}$. A point $P$ about $P_{0}$ is projected onto the tangent plane by (15). We assume that the cell containing the node $P_{0}$ is surrounded by $N_{0}$ hexagonal/ pentagonal cells and each surrounding cell has $N_{s}(s=$ $\left.1,2, \ldots, N_{0}\right)$ vertices. Here, we consider the spherical icosahedral mesh: $N_{0}=5$ or 6 and $N_{s}=5$ or 6 . The $j$ th vertex of the $i$ th cell is denoted by $Q_{i, j}\left(i=0,1, \ldots, N_{0}\right.$; $\left.j=1,2, \ldots, N_{i}\right)$ (Fig. A1). We indicate the midpoints of the arcs $P_{i} Q_{i, j}$ and $\widehat{Q_{i, j} Q_{i, j-1}}$ by $\mathbf{x}_{i, j}$ and $\mathbf{x}_{i, j+N_{i}}$, respectively, and the area of the spherical triangle $P_{i}$ $Q_{i, j-1} Q_{i, j}$ by $\alpha_{i, j}$. Note that $j-1$ is cyliclic, that is, it can be expressed as $1+$ modulo $\left(j-2, N_{i}\right)$ by the standard intrinsic procedure of FORTRAN 90. The cell mean value of $q$ is defined by $\bar{q}_{i}=\langle q\rangle_{i} / A_{i}$. Note that we have no interest in the location where $\bar{q}_{i}$ is defined. With these notations, the area integral of the quadratic polynomial

$\tilde{q}_{0}(x, y)=a_{0}+a_{1} x+a_{2} y+a_{3} x^{2}+a_{4} x y+a_{5} y^{2}$

over the $i$ th cell can be computed by

$$
\begin{aligned}
\int_{A_{i}} \tilde{q}_{0}(x, y) d A= & \left(a_{0}+a_{1} \bar{x}^{i}+a_{2} \bar{y}^{i}+a_{3}{\overline{x^{2}}}^{i}+a_{4} \overline{x y}^{i}\right. \\
& \left.+a_{5} \bar{y}^{2}{ }^{i}\right) A_{i}
\end{aligned}
$$

where

$\overline{x^{m} y^{n}}{ }^{i}=\frac{1}{A_{i}} \sum_{j=1}^{N_{i}} \frac{x_{i, j}^{m} y_{i, j}^{n}+x_{i, j-1}^{m} y_{i, j-1}^{n}+x_{i, j+N_{i}}^{m} y_{i, j+N_{i}}^{n}}{3} \alpha_{i, j}$

for $m=0,1,2$ and $n=0,1,2$. Note that $\mathbf{x}_{i, j}$ and $\mathbf{x}_{i, j+N_{i}}$ are the quadrature points of quadratic polynomials.

For the surrounding cells, we construct the following matrix formula

$$
\mathbf{q}^{\prime}=\mathbf{B a}
$$




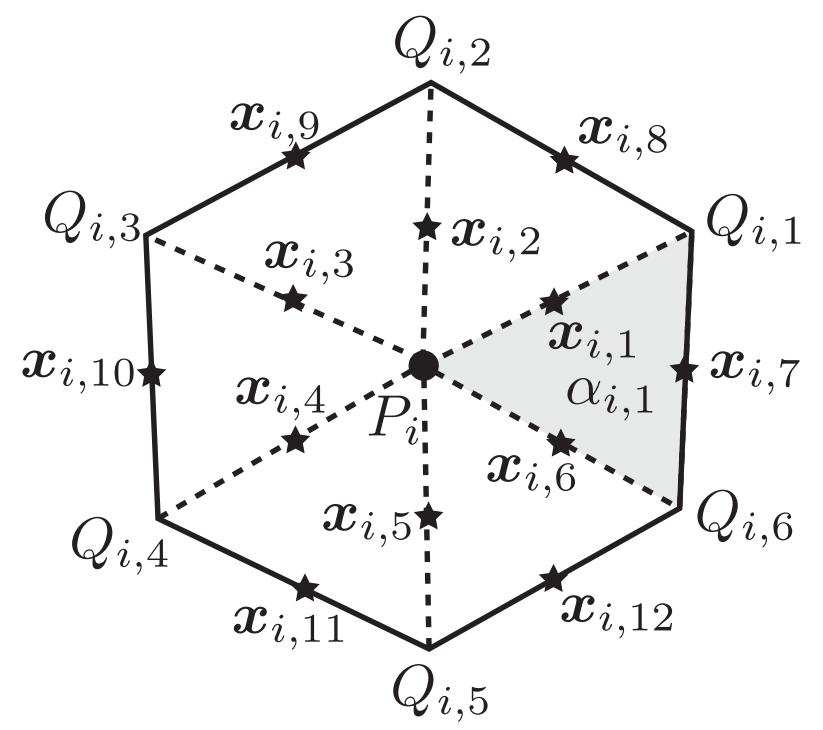

FIG. A1. A schematic illustration of the $i$ th hexagonal cell.

where

$$
\begin{aligned}
\mathbf{q}^{\prime} & =\left(\begin{array}{c}
\bar{q}_{1}-a_{0} \\
\vdots \\
\bar{q}_{N_{s}}-a_{0}
\end{array}\right), \\
\mathbf{B} & =\left(\begin{array}{ccccc}
\bar{x}^{1} & \bar{y}^{1} & \bar{x}^{2} & \overline{x y}^{1} & \bar{y}^{1} \\
\vdots & \vdots & \vdots & \vdots & \vdots \\
\bar{x}^{N_{s}} & \bar{y}^{N_{s}} & \bar{x}^{N^{2}} & \overline{x y}^{N_{s}} & \bar{y}^{N^{2}}
\end{array}\right), \quad \text { and } \\
\mathbf{a} & =\left(\begin{array}{c}
a_{1} \\
\vdots \\
a_{5}
\end{array}\right) \cdot
\end{aligned}
$$

The coefficient vector $\mathbf{a}$ is computed by inverting the matrix $\mathbf{B}$ as $\mathbf{a}=\mathbf{B}^{\#} \mathbf{q}^{\prime}$. Here, $\mathbf{B}^{\#}$ is the inverse matrix $\left(\mathbf{B}^{-1}\right)$ for pentagonal cells or the pseudoinverse matrix (B*) for hexagonal cells. We use the singular vector decomposition (SVD) algorithm (Golub and Reinsch 1970) to obtain $\mathbf{B}^{-1}$ or $\mathbf{B}^{*}$.

To close the derivation, we substitute the relation $\mathbf{a}=\mathbf{B}^{\#} \mathbf{q}^{\prime}$ into the area integral over the 0th cell to obtain

$$
\bar{q}_{0}=\left(\begin{array}{llll}
\beta_{0} & \beta_{1} & \ldots & \beta_{N_{s}}
\end{array}\right)\left(\begin{array}{c}
-a_{0} \\
\bar{q}_{1}-a_{0} \\
\vdots \\
\bar{q}_{N_{s}}-a_{0}
\end{array}\right),
$$

where

$$
\begin{aligned}
\left(\begin{array}{rlll}
\beta_{1} & \beta_{0} & \cdots & \beta_{N_{s}}
\end{array}\right)= & \left(\begin{array}{cccccc}
1 & \bar{x}^{0} & \bar{y}^{0} & \bar{x}^{2} & \overline{x y}^{0} & \bar{y}^{0}
\end{array}\right) \\
& \times\left(\begin{array}{cc}
-1 & 0 \\
0 & \mathbf{B}^{\#}
\end{array}\right) .
\end{aligned}
$$

By solving (A8), we determine $a_{0}$ as

$$
a_{0}=\frac{\sum_{j=0}^{N_{i}} \beta_{j} \bar{q}_{j}}{\sum_{j=0}^{N_{i}} \beta_{j}} .
$$

By substituting $a_{0}$ into $\mathbf{q}^{\prime}$, we compute the remaining coefficients from $\mathbf{a}=\mathbf{B}^{\#} \mathbf{q}^{\prime}$.

\section{APPENDIX B}

\section{A Form of the Flux Divergence for Pentagonal Cells}

For a pentagonal cell about $P_{0}$, we need to think of an overlap of five different systems, while we synchronize two systems (B1 and B2) for hexagonal cells. Equations (3) and (4) are changed for the sth system to

$\left.\frac{\partial h_{s}}{\partial t}\right|_{P_{0}}=-\left[\nabla_{s} \cdot\left(h_{s} \mathbf{v}_{s}\right)\right]_{P_{0}}-\frac{\sum_{i=1, . ., 5 ; i \neq s}\left(h_{s, P_{0}}-h_{i, P_{0}}\right)}{5 \tau_{h}}$,

where the index $s=1,2, \ldots, 5$ is cyclic (Fig. 8b). The prognostic variables are converted to $h=\left(h_{1}+h_{2}+\right.$ $\left.h_{3}+h_{4}+h_{5}\right) / 5$ and $h_{d, s}=h_{s}-\left(h_{s-2}+h_{s-1}+h_{s+1}+\right.$ $\left.h_{s+2}\right) / 4$, and (B1) is converted to

$$
\begin{aligned}
\left.\frac{\partial h}{\partial t}\right|_{P_{0}} & =-\frac{1}{5}\left[\sum_{s=1}^{5} \nabla_{s} \cdot\left(h_{s} \mathbf{v}_{s}\right)\right], \quad \text { and } \\
\left.\frac{\partial h_{d, s}}{\partial t}\right|_{P_{0}} & =-\left[\nabla_{s} \cdot\left(h_{s} \mathbf{v}_{s}\right)-\frac{1}{4} \sum_{i=1, ., 5 ; i \neq s} \nabla_{i} \cdot\left(h_{i} \mathbf{v}_{i}\right)\right]_{P_{0}}-\left[\frac{h_{d, s}}{\tau_{h}}\right]_{P_{0}}
\end{aligned}
$$

By taking infinitesimal limit of $\tau_{h}$, (B3) converges to $h_{d, s}=0$, and thus $h=h_{1}=\cdots=h_{5}$. From (B2), we obtain

$$
\frac{\partial\langle h\rangle_{0}}{\partial t}=-\frac{1}{5} \sum_{s=1}^{5} \int_{A_{0}} \nabla_{s} \cdot\left(h \mathbf{v}_{s}\right) d A
$$

instead of (20). The area integral of the flux-divergence is spatially discretized for each $s$ as 


$$
\begin{aligned}
\int_{A_{0}} \nabla_{s} \cdot\left(h \mathbf{v}_{s}\right) d A \approx & \sum_{i=s, s+1} \hat{h}_{i} l_{i} \mathbf{n}_{i} \cdot \mathbf{v}_{s}+\hat{h}_{s-1} l_{s-1} \mathbf{n}_{s-1} \cdot \mathbf{v}_{s-2} \\
& +\hat{h}_{s+2} l_{s+2} \mathbf{n}_{s+2} \cdot \mathbf{v}_{s+2}+\frac{1}{2} \hat{h}_{s-2} l_{s-2} \mathbf{n}_{s-2} \\
& \times\left(\mathbf{v}_{s-2}+\mathbf{v}_{s+2}\right)
\end{aligned}
$$

and (B4) becomes (28).

\section{REFERENCES}

Arakawa, A., and V. R. Lamb, 1977: Computational design of the basic dynamical processes of the UCLA general circulation model. Methods Comput. Phys. Adv. Res. Appl., 17, 173-265, https://doi.org/10.1016/B978-0-12-460817-7.50009-4.

Boccaletti, S., J. Kurths, G. Osipov, D. L. Valladares, and C. S. Zhou, 2002: The synchronization of chaotic systems. Phys. Rep., 366, 1-101, https://doi.org/10.1016/S0370-1573(02)00137-0.

Bonaventura, L., and T. Ringler, 2005: Analysis of discrete shallow-water models on geodesic Delaunay grids with C-type staggering. Mon. Wea. Rev., 133, 2351-2373, https://doi.org/ 10.1175/MWR2986.1.

Chen, C., J. Bin, F. Xiao, X. Li, and X. Shen, 2014: A global shallow-water model on an icosahedral-hexagonal grid by a multi-moment constrained finite-volume scheme. Quart. J. Roy. Meteor. Soc., 140, 639-650, https://doi.org/10.1002/qj.2157.

Cotter, C. J., and J. Shipton, 2012: Mixed finite elements for numerical weather prediction. J. Comput. Phys., 231, 7076-7091, https://doi.org/10.1016/j.jcp.2012.05.020.

Danilov, S., D. Sidorenko, Q. Wang, and T. Jung, 2017: The FinitevolumE Sea ice-Ocean Model (FESOM2). Geosci. Model Dev., 10, 765-789, https://doi.org/10.5194/gmd-10-765-2017.

Du, Q., M. D. Gunzburger, and L. Ju, 2003: Constrained centroidal Voronoi tessellations for surfaces. SIAM J. Sci. Comput., 24, 1488-1506, https://doi.org/10.1137/S1064827501391576.

Dubos, T., S. Dubey, M. Tort, R. Mittal, Y. Meurdesoif, and F. Hourdin, 2015: DYNAMICO-1.0, an icosahedral hydrostatic dynamical core designed for consistency and versatility. Geosci. Model Dev., 8, 3131-3150, https://doi.org/10.5194/ gmd-8-3131-2015.

Eldred, C., 2015: Linear and nonlinear properties of numerical methods for the rotating shallow water equations. Ph.D. dissertation, Colorado State University, $221 \mathrm{pp}$.

Fujisaka, H., and T. Yamada, 1983: Stability theory of synchronized motion in coupled-oscillator systems. Prog. Theor. Phys., 69, 32-47, https://doi.org/10.1143/PTP.69.32.

Galewsky, J., R. K. Scott, and L. M. Polvani, 2004: An initial-value problem for testing numerical models of the global shallowwater equations. Tellus, 56A, 429-440, https://doi.org/10.3402/ tellusa.v56i5.14436.

Golub, G. H., and C. Reinsch, 1970: Singular value decomposition and least squares solutions. Numer. Math., 14, 403-420, https:// doi.org/10.1007/BF02163027.

Hasegawa, M., and M. Tanemura, 1976: On the pattern of space division by territories. Ann. Inst. Stat. Math., 28, 509-519, https://doi.org/10.1007/BF02504768.

Heikes, R., and D. A. Randall, 1995: Numerical integration of the shallow-water equations on a twisted icosahedral grid. Part I: Basic design and results of tests. Mon. Wea. Rev., 123, 1862-1880, https:// doi.org/10.1175/1520-0493(1995)123<1862:NIOTSW>2.0.CO;2.
Jakob-Chien, R., J. J. Hack, and D. L. Williamson, 1995: Spectral transform solutions to the shallow water test set. J. Comput. Phys., 119, 164-187, https://doi.org/10.1006/jcph.1995.1125.

Majewski, D., and Coauthors, 2002: The Operational Global Icosahedral-Hexagonal Gridpoint Model GME: Description and high-resolution tests. Mon. Wea. Rev., 130, 319-338, https:// doi.org/10.1175/1520-0493(2002)130<0319:TOGIHG >2.0.CO;2.

Masuda, Y., and H. Ohnishi, 1986: An integration scheme of the primitive equation model with an icosahedral-hexagonal grid system and its application to the shallow water equations. J. Meteor. Soc. Japan, 64A, 317-326, https://doi.org/10.2151/ jmsj1965.64A.0_317.

Mesinger, D., 2000: Numerical methods: The Arakawa approach, horizontal grid, global, and limited-area modeling. General Circulation Model Development, D. A. Randall, Ed., Academic Press, 373-419.

Miura, H., 2004: Development of a mixed finite-difference/finitevolume scheme for the shallow water model on a spherical geodesic grid. Ph.D. dissertation, University of Tokyo, Tokyo, Japan, 205 pp.

_ 2013: An upwind-biased conservative transport scheme for multistage temporal integrations on spherical icosahedral grids. Mon. Wea. Rev., 141, 4049-4068, https://doi.org/10.1175/ MWR-D-13-00083.1.

_ 2017: Coupling the hexagonal B1-grid and B2-grid to avoid a computational mode problem of the hexagonal ZM-grid. SOLA, 13, 69-73, https://doi.org/10.2151/sola.2017-013.

_ , and M. Kimoto, 2005: A comparison of grid quality of optimized spherical hexagonal-pentagonal geodesic grids. Mon. Wea. Rev., 133, 2817-2833, https://doi.org/10.1175/MWR2991.1.

_ scheme using a quadratic reconstruction on spherical icosahedral grids. Mon. Wea. Rev., 141, 832-847, https://doi.org/ 10.1175/MWR-D-11-00355.1.

_ M. Satoh, T. Nasuno, A. T. Noda, and K. Oouchi, 2007: A Madden-Julian oscillation event realistically simulated by a global cloud-resolving model. Science, 318, 1763-1765, https:// doi.org/10.1126/science.1148443.

Miyamoto, Y., Y. Kajikawa, R. Yoshida, T. Yamaura, H. Yashiro, and H. Tomita, 2013: Deep moist atmospheric convection in a subkilometer global simulation. Geophys. Res. Lett., 40, 49224926, https://doi.org/10.1002/grl.50944.

Ničković, S., M. B. Gavrilov, and I. A. Tošić, 2002: Geostrophic adjustment on hexagonal grids. Mon. Wea. Rev., 130, 668-683, https:// doi.org/10.1175/1520-0493(2002)130<0668:GAOHG>2.0.CO;2.

Peixoto, P. S., 2016: Accuracy analysis of mimetic finite volume operators on geodesic grids and a consistent alternative. J. Comput. Phys., 310, 127-160, https://doi.org/10.1016/ j.jcp.2015.12.058.

Randall, D. A., 1994: Geostrophic adjustment and the finitedifference shallow-water equations. Mon. Wea. Rev., 122, 1371-1377, https://doi.org/10.1175/1520-0493(1994)122<1371: GAATFD $>2.0 . \mathrm{CO} ; 2$.

Ringler, T. D., and D. A. Randall, 2002a: A potential enstrophy and energy conserving numerical scheme for solution of the shallow-water equations on a geodesic grid. Mon. Wea. Rev., 130, 1397-1410, https://doi.org/10.1175/1520-0493(2002) $130<1397$ :APEAEC $>2.0 . \mathrm{CO} ; 2$.

— Mon. Wea. Rev., 130, 1411-1422, https://doi.org/10.1175/15200493(2002)130<1411:TZGAAT>2.0.CO;2.

_ J. Thuburn, J. B. Klemp, and W. C. Skamarock, 2010: A unified approach to energy conservation and potential vorticity 
dynamics for arbitrarily-structured C-grids. J. Comput. Phys., 229, 3065-3090, https://doi.org/10.1016/j.jcp.2009.12.007.

Rípodas, P., and Coauthors, 2009: Icosahedral Shallow Water Model (ICOSWM): Results of shallow water test cases and sensitivity to model parameters. Geosci. Model Dev., 2, 231251, https://doi.org/10.5194/gmd-2-231-2009.

Sadourny, R., A. Arakawa, and Y. Mintz, 1968: Integration of the nondivergent barotropic vorticity equation with an icosahedral-hexagonal grid for the sphere. Mon. Wea. Rev., 96, 351-356, https://doi.org/10.1175/1520-0493(1968)096<0351: IOTNBV $>2.0 . \mathrm{CO} ; 2$.

Satoh, M., T. Matsuno, H. Tomita, H. Miura, T. Nasuno, and S. Iga, 2008: Nonhydrostatic icosahedral atmospheric model (NICAM) for global cloud resolving simulations. J. Comput. Phys., 227, https://doi.org/10.1016/j.jcp.2007.02.006.

— mospheric Model: Description and development. Prog. Earth Planet. Sci., 1, 18, https://doi.org/10.1186/s40645-014-0018-1.

Shipton, J., T. H. Gibson, and C. J. Cotter, 2018: Higher-order compatible finite element schemes for the nonlinear rotating shallow water equations on the sphere. J. Comput. Phys., 375, 1121-1137, https://doi.org/10.1016/j.jcp.2018.08.027.

Skamarock, W. C., and A. Gassmann, 2011: Conservative transport schemes for spherical geodesic grids: High-order flux operators for ODE-based time integration. Mon. Wea. Rev., 139, 2962-2975, https://doi.org/10.1175/MWR-D-10-05056.1.

— J. B. Klemp, M. G. Duda, L. D. Fowler, S. Park, and T. D. Ringler, 2012: A multiscale nonhydrostatic atmospheric model using centroidal Voronoi tesselations and C-grid staggering. Mon. Wea. Rev., 140, 3090-3105, https://doi.org/10.1175/ MWR-D-11-00215.1.

Stuhne, G. R., and W. R. Peltier, 1996: Vortex erosion and amalgamation in a new model of large scale flow on the sphere. J. Comput. Phys., 128, 58-81, https://doi.org/10.1006/jcph.1996.0196.

Thuburn, J., 1995: Dissipation and cascades to small scales in numerical models using a shape-preserving advection scheme. Mon. Wea. Rev., 123, 1888-1903, https://doi.org/10.1175/15200493(1995)123<1888:DACTSS > 2.0.CO;2.

__, 1996: Multidimensional flux-limited advection schemes. J. Comput. Phys., 123, 74-83, https://doi.org/10.1006/ jcph.1996.0006.

—- and Y. Li, 2000: Numerical simulations of RossbyHaurwitz waves. Tellus, 52A, 181-189, https://doi.org/ 10.3402/tellusa.v52i2.12258.

— , and C. J. Cotter, 2015: A primal-dual mimetic finite element scheme for the rotating shallow water equations on polygonal spherical meshes. J. Comput. Phys., 290, 274-297, https:// doi.org/10.1016/j.jcp.2015.02.045.

_ T. D. Ringler, W. C. Skamarock, and J. B. Klemp, 2009: Numerical representation of geostrophic modes on arbitrarily structured C-grids. J. Comput. Phys., 228, 8321-8335, https:// doi.org/10.1016/j.jcp.2009.08.006.

Tomita, H., M. Tsugawa, M. Satoh, and K. Goto, 2001: Shallow water model on a modified icosahedral geodesic grid by using spring dynamics. J. Comput. Phys., 174, 579-613, https:// doi.org/10.1006/jcph.2001.6897.

— M. Satoh, and K. Goto, 2002: An optimization of the icosahedral grid modified by spring dynamics. J. Comput. Phys., 183, 307-331, https://doi.org/10.1006/jcph.2002.7193.

Ullrich, P. A., C. Jablonowski, and B. van Leer, 2010: High-order finite-volume methods for the shallow-water equations on the sphere. J. Comput. Phys., 229, 6104-6134, https://doi.org/ 10.1016/j.jcp.2010.04.044.

-, and Coauthors, 2017: DCMIP2016: A review of nonhydrostatic dynamical core design and intercomparison of participating models. Geosci. Model Dev., 10, 4477-4509, https://doi.org/10.5194/gmd-10-4477-2017.

Walko, R. L., and R. Avissar, 2008a: The Ocean-LandAtmosphere Model (OLAM). Part I: Shallow-water tests. Mon. Wea. Rev., 136, 4033-4044, https://doi.org/10.1175/ 2008MWR2522.1.

- , and - , 2008b: The Ocean-Land-Atmosphere Model (OLAM). Part II: Formulation and tests of the nonhydrostatic dynamic core. Mon. Wea. Rev., 136, 4045-4062, https://doi.org/ 10.1175/2008MWR2523.1.

Weller, H., J. Thuburn, and C. J. Cotter, 2012: Computational modes and grid imprinting on five quasi-uniform spherical C grids. Mon. Wea. Rev., 140, 2734-2755, https://doi.org/10.1175/ MWR-D-11-00193.1.

Wicker, L. J., and W. C. Skamarock, 2002: Time-splitting methods for elastic models using forward time schemes. Mon. Wea. Rev., 130, 2088-2097, https://doi.org/10.1175/1520-0493(2002) $130<2088$ :TSMFEM $>2.0 . \mathrm{CO} ; 2$.

Williamson, D. L., 1968: Integration of the barotropic vorticity equation on a spherical geodesic grid. Tellus, 20, 642-653, https://doi.org/10.3402/tellusa.v20i4.10044.

__ 1970: Integration of the primitive barotropic model over a spherical geodesic grid. Mon. Wea. Rev., 98, 512-520, https://doi.org/10.1175/1520-0493(1970)098<0512:IOTPBM > 2.3.CO;2.

—_, J. B. Drake, J. J. Hack, R. Jakob, and P. N. Swarztrauber, 1992: A standard test set for numerical approximations to the shallow water equations in spherical geometry. J. Comput. Phys., 102, 211-224, https://doi.org/10.1016/ S0021-9991(05)80016-6.

Zängl, G., D. Reinert, P. Rípodas, and M. Baldauf, 2015: The ICON (ICOsahedral Non-hydrostatic) modelling framework of DWD and MPI-M: Description of the non-hydrostatic dynamical core. Quart. J. Roy. Meteor. Soc., 141, 563-579, https:// doi.org/10.1002/qj.2378. 